\title{
A poroelastic fluid-structure interaction model of syringomyelia
}

DOI:

10.1017/jfm.2016.669

Document Version

Accepted author manuscript

Link to publication record in Manchester Research Explorer

\section{Citation for published version (APA):}

Heil, M., \& Bertram, C. D. (2016). A poroelastic fluid-structure interaction model of syringomyelia. Journal of Fluid Mechanics, 809, 360-389. https://doi.org/10.1017/jfm.2016.669

\section{Published in:}

Journal of Fluid Mechanics

\section{Citing this paper}

Please note that where the full-text provided on Manchester Research Explorer is the Author Accepted Manuscript or Proof version this may differ from the final Published version. If citing, it is advised that you check and use the publisher's definitive version.

\section{General rights}

Copyright and moral rights for the publications made accessible in the Research Explorer are retained by the authors and/or other copyright owners and it is a condition of accessing publications that users recognise and abide by the legal requirements associated with these rights.

\section{Takedown policy}

If you believe that this document breaches copyright please refer to the University of Manchester's Takedown Procedures [http://man.ac.uk/04Y6Bo] or contact uml.scholarlycommunications@manchester.ac.uk providing relevant details, so we can investigate your claim.

\section{OPEN ACCESS}




\title{
A poroelastic fluid-structure interaction model of syringomyelia
}

\author{
By Matthias Heil ${ }^{1} \dagger$ AND Christopher D. Bertram ${ }^{2}$ \\ ${ }^{1}$ School of Mathematics, University of Manchester, Oxford Road, Manchester, M13 9PL, UK \\ ${ }^{2}$ School of Mathematics and Statistics, University of Sydney, NSW 2006, Australia
}

(Received ?; revised ?; accepted ?. - To be entered by editorial office)

Syringomyelia is a medical condition in which one or more fluid-filled cavities (syrinxes) form in the spinal cord. The syrinxes often form near locations where the spinal subarachnoid space (SSS; the fluid-filled annular region surrounding the spinal cord) is partially obstructed. Previous studies showed that nonlinear interactions between the pulsatile fluid flow in the SSS and the elastic deformation of the tissues surrounding it can generate a fluid pressure distribution that would tend to drive fluid from the SSS into the syrinx if the tissue separating the two regions was porous. This provides a potential explanation for why a partial occlusion of the SSS can induce the growth of an already existing nearby syrinx. We study this hypothesis by analysing the mass transfer between the SSS and the syrinx, using a poroelastic fluid-structure interaction model of the spinal cord that includes a representation of the partially obstructed SSS, the syrinx and the poroelastic tissues surrounding these fluid-filled cavities. Our numerical simulations show that poroelastic fluid-structure interaction can indeed cause an increase (albeit relatively small) in syrinx volume. We analyse the seepage flows and show that their structure can be captured by an analytical model which explains why the increase in syrinx volume tends to be relatively small.

\section{Introduction}

The brain and the spinal cord are submerged in cerebrospinal fluid (CSF), a water-like substance that occupies the subarachnoid space and the brain's ventricles. MRI scans show evidence of oscillatory flows along the spinal subarachnoid space (SSS), the annular CSF-filled region that surrounds the spinal cord. These flows are driven by the timeperiodic systolic expansion of the intracranial extracerebral arteries which displaces CSF from the brain into the SSS. The SSS accommodates the fluid by increasing its volume via the compression of the spinal cord and the expansion of the dura, the tissue forming the outer boundary of the SSS; see Fig. 1 below for a schematic showing the geometry. Typical amplitudes of the resulting pulsatile fluid velocities are in the region of $5-10 \mathrm{~cm} \mathrm{~s}^{-1}$; see, e.g., Hentschel et al. (2010) or Bunck et al. (2011). The ability to alleviate pressure fluctuations in the brain via the expansion of the SSS can be severely compromised by conditions such as Chiari malformation which form an obstruction between the cranial and spinal subarachnoid spaces.

The pressure fluctuations associated with the pulsatile flow of CSF along the elastically deforming SSS have long been suspected of playing a possible role in the pathogenesis of syringomyelia - a medical condition in which one or more fluid-filled cavities (so-called

$\dagger$ Email address for correspondence: M.Heil@maths.manchester.ac.uk 
syrinxes) form in the spinal cord. These syrinxes often grow over time and ultimately cause serious medical problems; see Elliott et al. (2013) for a detailed recent review. Syrinxes often form near regions where the SSS is partially obstructed, for instance by Chiari malformations (see, e.g., Shaffer et al. 2011) or as a result of injuries arising from spinal trauma. This raises the question of whether such obstructions affect the flow of CSF in such a way that the formation of syrinxes is facilitated. This question motivated early theoretical studies by Carpenter and co-workers (Berkouk et al. 2003, Carpenter et al. 2003; also Cirovic 2009 and Cirovic \& Kim 2012) who employed spatially onedimensional models to analyse the propagation of large-amplitude pressure waves along the elastic-walled SSS. These studies were based on extensions of the classical theory of pulse-wave propagation in the arteries (see van de Vosse \& Stergiopulos (2011) for a review), combining $1 \mathrm{D}$ inviscid fluid models with so-called tube-laws which describe the change in the SSS's local cross-sectional area as a function of the local fluid pressure. One early conjecture was that the large pressures associated with the formation of "elastic jumps" generated by the presence of partial obstructions in the SSS could cause damage to the cord tissue. This hypothesis was later discredited by Bertram et al. (2005) who were the first to analyse the system via the coupled solution of the axisymmetric NavierStokes equations and the equations of elasticity; Elliott et al. (2009) later reached the same conclusions on the basis of a $1 \mathrm{D}$ model.

Bertram et al.'s (2005) model was subsequently refined by including a representation of (i) a syrinx (Bertram 2009) and (ii) an obstruction of the SSS (Bertram 2010), resulting in the model shown in Fig. 1 below. Bertram's (2010) numerical simulations of this system and Martin et al.'s (2010) experimental study of a related benchtop model showed that when this system is subjected to time-periodic pressure fluctuations at its cranial end, nonlinear effects arising from fluid-structure interaction (FSI) lead to an increase in the CSF pressure in the caudal part of the SSS via what Martin et al. (2010) termed the "valve mechanism". Bertram (2010) observed that the resulting CSF pressure distribution was such that it would tend to drive fluid from the SSS into the syrinx if the cord tissue was porous. This provides a possible explanation for why the presence of an obstruction in the SSS may induce the growth of an adjacent syrinx: the inflation of the syrinx may provide a stimulus for the subsequent remodelling of the tissue to relieve the induced mechanical stresses.

It is the aim of the present paper to assess whether the incorporation of poroelasticity into Bertram's (2010) fluid-structure interaction model does indeed result in an increase in the syrinx volume via Martin et al.'s (2010) "valve mechanism". The outline of the paper is as follows. In $\S 2$ we discuss our theoretical model which is based on the coupled solution of the axisymmetric Navier-Stokes equations and the equations of isotropic poroelasticity. Following a brief discussion of the numerical solution in $\S 3$ we present our results in $\S 4$. We show that the "valve mechanism" observed in the impermeable system persists in the presence of poroelasticity and that the resulting seepage flows do indeed result in an increase (albeit relatively small) in syrinx volume. We analyse the seepage flows and show that their structure can be captured by an analytical model which also explains how the system evolves towards its ultimate time-periodic steady state and why the increase in syrinx volume is very small. Finally, in $\S 5$ we provide a brief summary of our results and assess the limitations of our analysis by discussing various features that are not represented in our model. 


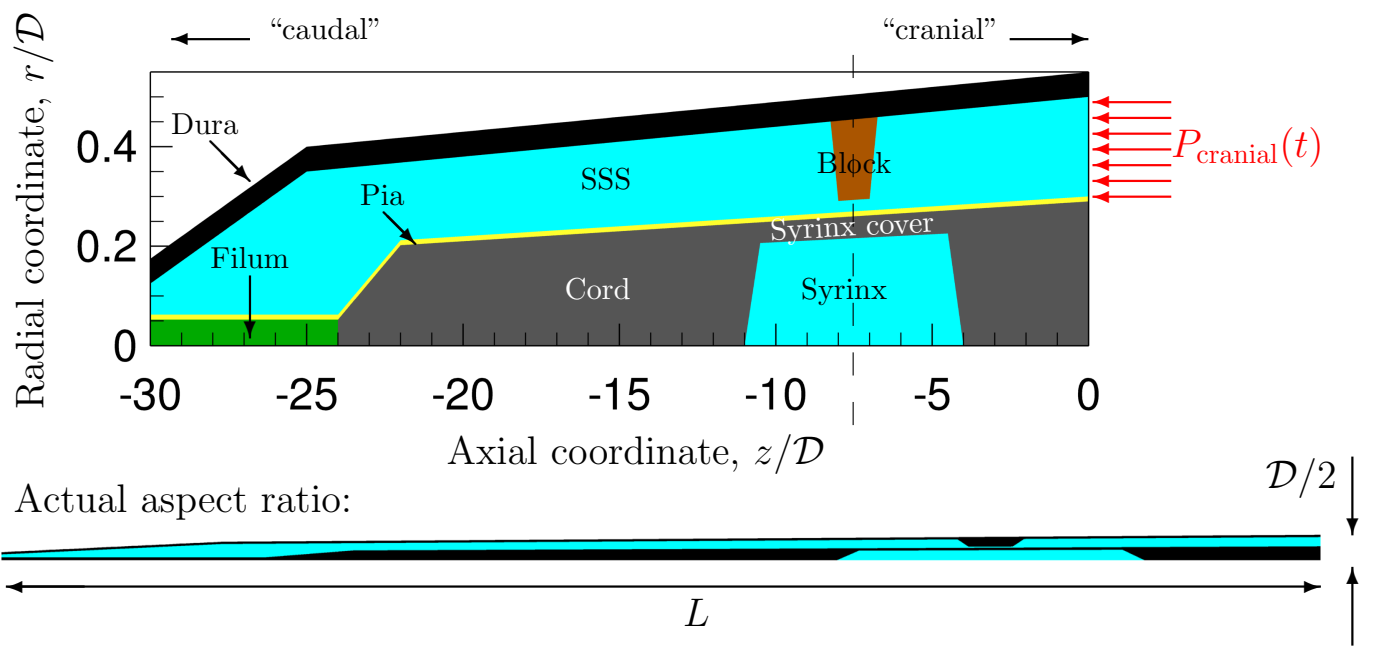

FIGURE 1. Sketch of the axisymmetric model. Cerebrospinal fluid (CSF; cyan) occupies the annular spinal subarachnoid space (SSS) which is partly occluded by a blockage. The syrinx is the fluid-filled cavity inside the spinal cord. In the top figure different colours identify the different tissues; see text for details. The fluid occupying the SSS is subject to an oscillatory pressure at the cranial end of the SSS. Note the large aspect ratio, $L / \mathcal{D}=30$, where $\mathcal{D}=20 \mathrm{~mm}$ is the maximum outer diameter of the SSS (at its cranial end). The dashed line indicates the position of the radial slice across which we compute the volume fluxes that are documented in Fig. 2.

\section{The model}

Fig. 1 shows a sketch of Bertram's (2010) axisymmetric model which forms the basis of the current study. CSF (cyan) is contained in the annular SSS the outer boundary of which is formed by the stiff dura (black). The spinal cord (gray) is a relatively soft tissue that is surrounded by a thin outer layer of increased stiffness, the pia (yellow). The cord ends in the filum (green). The syrinx is the fluid-filled cavity (cyan) inside the cord. A "block" (brown) forms a partial occlusion of the SSS near the syrinx. We refer to the thin layer of tissue that separates the SSS from the syrinx as the syrinx cover. The system is subject to a time-periodic pressure, $P_{\text {cranial }}(t)$, that is imposed at the cranial end of the SSS (at $z=0$ ) and we neglect the effect of gravity.

We assume that the fluid occupying the SSS and the syrinx is incompressible, and that its velocity $\mathbf{v}$ and pressure $p$ are governed by the Navier-Stokes equations,

$$
\rho_{\text {fluid }}\left(\frac{\partial \mathbf{v}}{\partial t}+\mathbf{v} \cdot \nabla \mathbf{v}\right)=\nabla \cdot \boldsymbol{\tau} \quad \text { and } \quad \nabla \cdot \mathbf{v}=0,
$$

where $\rho_{\text {fluid }}$ is the fluid density. Assuming Newtonian behaviour, the fluid stress tensor $\tau$ is given by

$$
\boldsymbol{\tau}=-p \mathbf{I}+\mu_{\text {fluid }}\left(\nabla \mathbf{v}+(\nabla \mathbf{v})^{T}\right),
$$

where $\mu_{\text {fluid }}$ is the fluid's dynamic viscosity.

We describe the deformation of the various elastic and poroelastic tissues and the 
seepage flow within the latter in terms of the displacement $\mathbf{u}$ of their solid matrix, the seepage flow $\mathbf{q}$ (relative to the deforming solid matrix), and the pore pressure, $p_{\text {pore }}$. The poroelastic tissues are assumed to be fully saturated with pore fluid. Using Biot's classical isotropic linear theory of poroelasticity (see, e.g. Simon (1992) or Cowin (1999)), these fields are governed by the momentum equation for the compound poroelastic material,

$$
\nabla \cdot \boldsymbol{\sigma}=\rho_{\text {p.e. }} \frac{\partial^{2} \mathbf{u}}{\partial t^{2}}+\rho_{\text {fluid }} \frac{\partial \mathbf{q}}{\partial t}
$$

and the momentum equation for the pore fluid (Darcy's law),

$$
-\nabla p_{\text {pore }}-\frac{\mu_{\text {fluid }}}{k} \mathbf{q}=\rho_{\text {fluid }} \frac{\partial^{2} \mathbf{u}}{\partial t^{2}}+\frac{\rho_{\text {fluid }}}{\phi} \frac{\partial \mathbf{q}}{\partial t} .
$$

Here $k$ is the permeability, $\phi$ the porosity (the volume fraction occupied by the pore space), and

$$
\rho_{\text {p.e. }}=(1-\phi) \rho_{\text {solid }}+\phi \rho_{\text {fluid }}
$$

is the density of the compound, poroelastic medium which comprises the solid matrix (which is made of material that has density $\rho_{\text {solid }}$ ) and the embedded pore fluid. The terms on the right-hand-side of equations (2.3) and (2.4) represent inertial effects. These arise from the accelerations of the solid matrix and the CSF fluid that flows within (and relative to) that matrix. Neglecting these terms in equation (2.4) we recover the classical, quasi-steady Darcy model. The stress in the compound poroelastic material is given by the constitutive equation

$$
\boldsymbol{\sigma}=\boldsymbol{\sigma}_{\text {elast }}-\alpha p_{\text {pore }} \mathbf{I}
$$

where

$$
\boldsymbol{\sigma}_{\text {elast }}=\frac{E_{\text {drained }}}{\left(1+\nu_{\text {drained }}\right)}\left(\frac{\nu_{\text {drained }}}{\left(1-2 \nu_{\text {drained }}\right)}(\nabla \cdot \mathbf{u}) \mathbf{I}+\frac{1}{2}\left(\nabla \mathbf{u}+(\nabla \mathbf{u})^{T}\right)\right)
$$

is the stress tensor for the drained solid matrix in terms of its Young's modulus, $E_{\text {drained }}$, Poisson's ratio, $\nu_{\text {drained }}$, and the Biot-Willis parameter, $\alpha$.

Mass conservation requires that

$$
\alpha \nabla \cdot\left(\frac{\partial \mathbf{u}}{\partial t}\right)+\nabla \cdot \mathbf{q}=0
$$

so the dynamic compression/dilation of the solid matrix acts as source/sink term in the continuity equation for the pore fluid. The Biot-Willis parameter $\alpha$ "represents the ratio of the fluid volume gained (or lost) in a material element to the volume change of that element, when the pore pressure is allowed to return to its initial state" (Detournay \& Cheng 1993). $\alpha$ is equal to one if the pore fluid and the material making up the solid matrix are both incompressible. Purely elastic (impermeable) behaviour can be recovered by setting the Biot-Willis parameter to $\alpha=0$, and by imposing $\mathbf{q}=\mathbf{0}$ and $p_{\text {pore }}=0$.

\subsection{Boundary conditions}

Equations (2.3)-(2.8) apply throughout the various tissues (cord, pia, etc) which have different mechanical properties as reflected in the values of their constitutive parameters, discussed in $\S 2.2$ below. At the material interfaces between the tissues we impose continuity of traction, displacement, pore pressure and the normal component of the seepage velocity.

We set the normal component of the traction acting on the fluid at the cranial end of 
the SSS to the imposed oscillatory pressure, $P_{\text {cranial }}(t)$, so that

$$
-\mathbf{e}_{z} \cdot \boldsymbol{\tau} \cdot \mathbf{e}_{z}=P_{\text {cranial }}(t) \quad \text { at } z=0,
$$

and enforce parallel inflow/outflow,

$$
\mathbf{v} \cdot \mathbf{e}_{r}=0 \quad \text { at } z=0 .
$$

At the caudal end of the SSS we suppress any inflow/outflow by setting

$$
\mathbf{v} \cdot \mathbf{e}_{z}=0 \quad \text { at } z=-L .
$$

We constrain the axial motion of the dura and the cord at the cranial and caudal ends of the domain (so that $\mathbf{u} \cdot \mathbf{e}_{z}=0$ at $z=0$ and $z=-L$ ) but allow the tissues to expand freely in the radial direction.

At the FSI interfaces, fluid and solid interact via the continuity of traction and velocity/displacements; see, e.g., Badia et al. (2009). The compound poroelastic solids are subject to the fluid traction, implying that

$$
\tau \cdot \mathbf{n}=\sigma \cdot \mathbf{n},
$$

where $\mathbf{n}$ is the outer unit normal on the poroelastic solid, pointing into the fluid. Furthermore, the pore pressure is given by the normal component of the fluid traction,

$$
p_{\text {pore }}=-\mathbf{n} \cdot \boldsymbol{\sigma} \cdot \mathbf{n} \text {. }
$$

The normal component of the fluid velocity at the FSI interfaces has to match the normal component of the velocity of the poroelastic solid plus the seepage flow,

$$
\mathbf{v} \cdot \mathbf{n}=\left(\frac{\partial \mathbf{u}}{\partial t}+\mathbf{q}\right) \cdot \mathbf{n} .
$$

Finally, the Beavers-Joseph-Saffman (BJS) boundary condition (Beavers \& Joseph 1967, Saffman 1971; see also Carraro et al. 2013) requires that the tangential component of the fluid velocity be equal to the tangential component of the velocity of the poroelastic solid plus a slip component,

$$
\mathbf{v} \cdot \mathbf{t}=\frac{\partial \mathbf{u}}{\partial t} \cdot \mathbf{t}-\frac{\sqrt{k}}{\gamma} \mathbf{t} \cdot \boldsymbol{\tau} \cdot \mathbf{n}
$$

where $\mathbf{t}$ is a unit tangent vector to the fluid-solid interface. Thus the slip velocity is proportional to the tangential shear stress exerted by the fluid onto the poroelastic solid; the slip vanishes as the inverse slip rate coefficient, $\gamma^{-1}$, goes to zero.

\subsection{Parameter values}

The spatial dimensions of the model were chosen to be identical to those of Bertram (2010), with an overall length of $L=60 \mathrm{~cm}$ and a maximum outer diameter of the SSS (at the cranial end) of $\mathcal{D}=20 \mathrm{~mm}$; see Fig. 1(b) for a plot of the system using a 1:1 aspect ratio. We refer to Bertram (2010) for full details of the geometry. As in Bertram (2010) we set the fluid and solid densities to $\rho_{\text {fluid }}=\rho_{\text {solid }}=1000 \mathrm{~kg} \mathrm{~m}^{-3}$, and assumed the dynamic viscosity of the CSF to be close to that of water, $\mu_{\text {fluid }}=10^{-3} \mathrm{~Pa}$ s. The dura and pia are much stiffer than the other tissues and, again following Bertram (2010), we set their (drained) Young's moduli to $E_{\text {drained }}^{[\text {dura }}=E_{\text {drained }}^{[\text {pia] }}=1.25 \times 10^{6} \mathrm{~Pa}$ while using $E_{\text {drained }}^{\text {[cord }}=5 \times 10^{3} \mathrm{~Pa}$ and $E_{\text {drained }}^{[\text {filum }]}=E_{\text {drained }}^{\text {[block }]}=6.25 \times 10^{4} \mathrm{~Pa}$ for the other tissues.

Since we are mainly interested in the fluid transport between the SSS and the syrinx, we only considered the cord tissue (including the pia and the filum) to be porous. For 
simplicity we assumed that the poroelastic parameters were the same throughout the pia, filum and cord. We used a a porosity of $\phi=0.3$ (Linninger et al. 2007), a default permeability of $k=10^{-13} \mathrm{~m}^{2}$, set the Biot-Willis parameter to $\alpha=1$ and the drained Poisson's ratio to $\nu_{\text {drained }}=0.35$ (Smillie et al. 2005, Tully \& Ventikos 2011). Extensive parameter studies were performed to assess the effect of variations in the permeability; see $\S 4$. The porosity $\phi$ only affects one of the inertial terms in (2.4) which, for the permeabilities and forcing frequencies considered in the present study were found to be negligible; see $\S 4.2$. The block and the dura were modelled as impermeable, nearincompressible solids, as in Bertram (2010), by setting the Biot-Willis parameter to $\alpha=0$, the Poisson's ratio to $\nu_{\text {[drained] }}=0.49$ and by imposing $\mathbf{q}=\mathbf{0}$ and $p_{\text {pore }}=0$ throughout these tissues. The inverse slip coefficient, $\gamma^{-1}$, in the BJS boundary condition (2.15) was set to zero after confirming that it had no effect on the results; see Fig. 13(b) in $\S 4.2$.

Simulations were performed using the same protocol employed in Bertram's (2010) study of the impermeable system. Starting from an initial condition in which the fluid and the solids were at rest (and the solids in their stress-free, undeformed configuration), we imposed an oscillatory pressure at the cranial end of the SSS,

$$
P_{\text {cranial }}(t)=\left\{\begin{array}{lr}
\widehat{\mathcal{P}} \sin ^{2}(2 \pi t / \mathcal{T}) & \text { for } t<\mathcal{T} / 4 \\
\widehat{\mathcal{P}} \sin (2 \pi t / \mathcal{T}) & \text { for } t \geq \mathcal{T} / 4,
\end{array}\right.
$$

and simulated the system's behaviour for at least ten periods of the oscillation, $0<t<$ $10 \mathcal{T}$. Following Bertram (2010), we set the peak pressure to $\widehat{P}=500 \mathrm{~Pa}$ and chose the period of the oscillation as $\mathcal{T}=0.4 \mathrm{~s}$.

When presenting results we generally non-dimensionalise all quantities on problemspecific reference values: time on the period of the oscillations, $\mathcal{T}$; lengths on the maximum outer diameter of the SSS, $\mathcal{D}$ (as in Fig. 1); time-varying volumes on their initial values at $t=0$; pressures on the peak forcing pressure, $\widehat{P}$; velocities on the associated inertial velocity scale, $\mathcal{U}=\left(\widehat{P} / \rho_{\text {fluid }}\right)^{1 / 2}$; and volume fluxes on $\mathcal{U} / \mathcal{D}^{2}$. It is important to note that the large nominal Reynolds number $R e_{\text {nominal }}=\rho_{\text {fluid }} \mathcal{U D} / \mu_{\text {fluid }}=14,142$ formed from these quantities does not adequately describe the character of the flow. This is because the CSF only flows in the thin annular SSS (or in the even narrower gap under the block), and/or with peak velocities that are significantly smaller than $\mathcal{U}$. The effective local Reynolds number, formed from the actual velocities and lengthscales (as illustrated in Fig. 4 below, for instance), tends to reach peak values of about 170, consistent with estimates obtained by Loth et al. (2001) based on MRI images of the CSF flow in actual spinal cavities.

\section{Numerical solution}

The governing equations were discretised by finite elements and implemented in a fully coupled manner in oomph-lib (Heil \& Hazel 2006). We discretised the arbitrary Lagrangian-Eulerian form of the Navier-Stokes equations using six-noded triangular Taylor-Hood elements on a moving unstructured mesh. Nodal positions in the fluid mesh were updated by the Method of Spines (Kistler \& Scriven 1983) by displacing fluid nodes such that they were always located at fixed fractions along straight lines that connected pre-determined material reference points on the moving FSI boundaries. The traction boundary condition (2.9) was incorporated into the boundary term that arises when integrating the weak form of the axial momentum equation by parts. The equations of poroelasticity were also discretised on six-noded triangles, using an isoparametric interpolation for the solid displacements, $\mathbf{u}$; second-order Raviart Thomas basis functions 
for the seepage flux, q; and piecewise linear, discontinuous basis functions for the pore pressure $p_{\text {pore }}$. See, e.g., Ervin $(2012,2013)$ for details. The FSI traction boundary conditions (2.12) and (2.13) were incorporated into the weak form of equations (2.3) and (2.4), respectively, while the coupling conditions (2.14) and (2.15) were imposed on the fluid velocities by Lagrange multipliers. First- and second-order time-derivatives were discretised using the implicit, second-order-accurate backward differencing (BDF2) and Newmark schemes, respectively. The resulting large system of nonlinear algebraic equations arising at each time-step was solved by Newton's method using the multifrontal direct solver MUMPS (Amestoy et al. 2001) to solve the associated linear systems. All meshes were generated with Shewchuk's (1996) Triangle code, with element sizes carefully adjusted to resolve the various boundary layers in the fluid and solid domains. The standard spatial discretisation involved 1.5 million degrees of freedom and time-stepping was performed with a fixed time-step corresponding to 80 time-steps per period of the imposed oscillation in $P_{\text {cranial }}(t)$. Results of spatial and temporal convergence tests and details of the code validation are presented in Appendix A.

\section{Results}

Fig. 2 shows a plot of the imposed pressure, $P_{\text {cranial }}(t)=p(z=0, t)$, and illustrates the resulting redistribution of cerebrospinal fluid along the SSS and within the syrinx, using time traces of the volume fluxes through (i) the cranial end of the SSS (at $z=0$ ); (ii) the narrow gap between the block and the cord (at $z / \mathcal{D}=-7.5$ ); (iii) a radial slice through the syrinx at the same $z$-position. (The location of the radial slice is indicated by the dashed line in Fig. 1). The volume flux is counted as positive when it is directed into the SSS, i.e. away from its cranial end. The plot shows that, following the decay of brief initial transients, the system settles into an approximately time-periodic response, with the inflow at the cranial end of the SSS being approximately $46^{\circ}$ out of phase with the driving pressure, suggesting that the system's input impedance arises through a combination of inertial, viscous and elastic effects. About $50 \%$ of the incoming volume flux passes through the narrow gap under the block into the caudal part of the SSS (dashed line in Fig. 2(b)) while the majority of the remaining volume flux is accommodated by the expansion of the cranial part of the SSS (the seepage flow into the porous cord is too small to be observable in these plots). Bertram's (2010) simulations of the impermeable system showed the presence of a noticeable time-periodic axial "sloshing flow" along the syrinx. This flow is also present in the permeable system; its magnitude is indicated by the plot of the volume flux across the radial slice through the syrinx (dash-dotted line in Fig. 2(b)).

The four panels in Fig. 3 provide a more detailed picture of the fluid-structure interaction when the system has settled into its steady state oscillation. In each of the panels, the bottom-most figure shows the fluid pressure distribution (represented by the colour contours) and the resulting solid deformation, using the system's actual aspect ratio. The middle and top figures show the same data but with all radial dimensions increased by a factor of 10. Finally, in the top figure the solid displacements are magnified by a factor of 20 to illustrate the deformation more clearly. The periodic expansion and contraction of the cranial part of the SSS which, as discussed above, accommodates a large fraction of the incoming flow, is clearly visible. The flow through the narrow gap between the block and the cord creates a large viscous pressure drop, the magnitude of which depends on the instantaneous width of the gap.

The contour plots in Fig. 4(a,b) show the fluid velocity field in the SSS in the vicinity of the block at two instants when the flow is driven most rapidly towards the cranial/caudal 
(a)

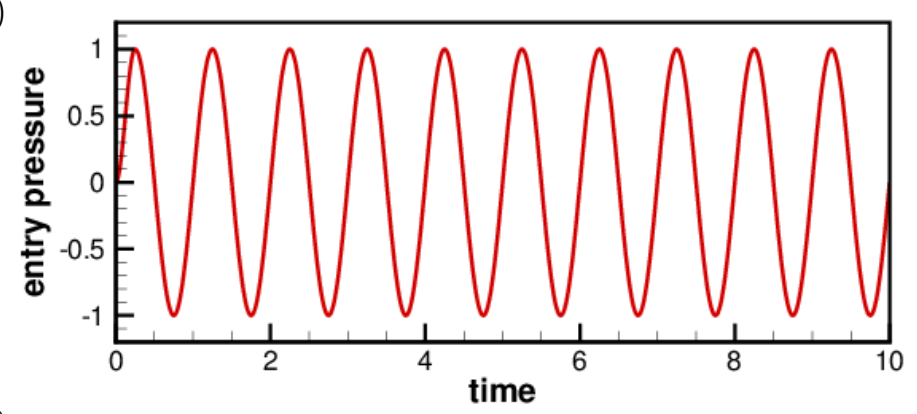

(b)

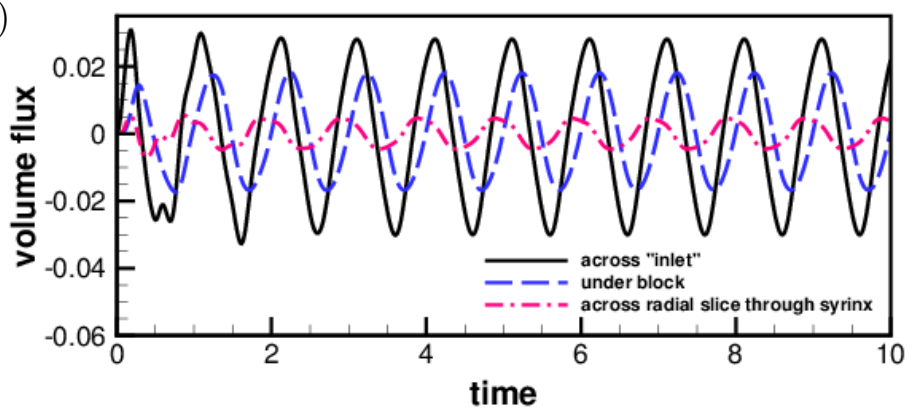

Figure 2. (a) Time trace of the non-dimensional forcing pressure, $P_{\text {cranial }}(t) / \widehat{\mathcal{P}}$. (b) The resulting volume flux into the SSS at $z=0$ (black solid); through the narrow gap under the block at $z / \mathcal{D}=-7.5$ (blue dashed); through a radial slice across the syrinx at the same axial position (red dash-dotted). The location of the radial slice is indicated by the dashed line in Fig. 1. Time is non-dimensionalised on the period of the oscillation, $\mathcal{T}$. The volume fluxes are scaled on $\mathcal{U D}^{2}$ where $\mathcal{U}=\left(\widehat{\mathcal{P}} / \rho_{\text {fluid }}\right)^{1 / 2}$ is the inertial velocity scale. The volume flux is positive when it is directed into the SSS, i.e. away from its cranial end.

ends of the SSS, respectively. Within the narrow gap between the block and the cord, the flow is approximately unidirectional, with a parabolic profile (see Fig. 4(c)) and the pressure decreasing linearly in the direction of the flow (see also Fig. 5 below). This indicates that, despite the large velocities, the flow in this region is of lubrication type and is therefore dominated by viscous effects. As the flow emerges from the narrow gap and decelerates, it separates off the solid boundaries. This results in the formation of strong transient vortices which are advected with the flow. Since the flow changes direction periodically, the vortices remain located in the vicinity of the block; their remnants remain visible even after the flow has reversed direction (see, e.g., the velocity perturbations at the cranial [or caudal] end of the block in Fig. 4(a) [or (b)], respectively. Away from the block, the velocity becomes approximately unidirectional again, but now with a Womersley profile (i.e. an inviscid plug flow profile in the centre of the SSS, with Stokes layers near the walls; see Fig. 4(d)), indicating that the flow in these regions is inertially dominated. In dimensional terms, the maximum oscillatory velocity in the profiles shown 
(a)

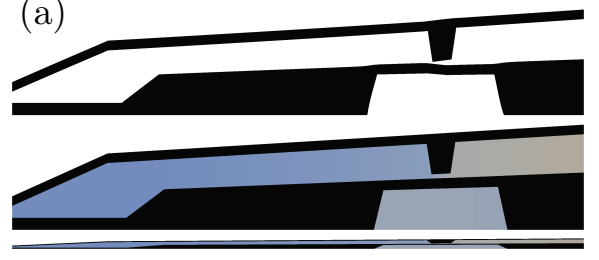

(c)

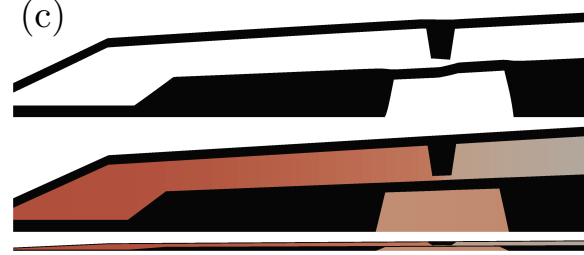

(b)

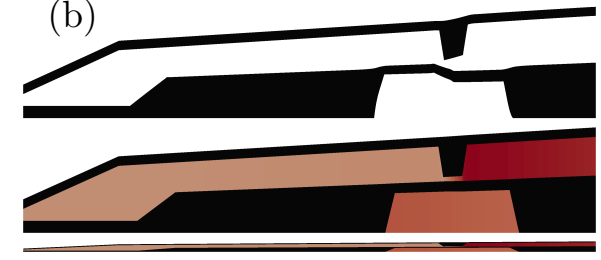

(d)

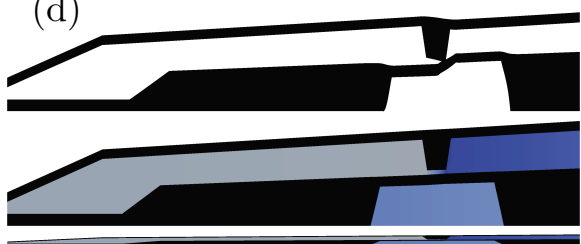

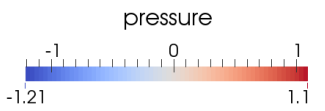

FIgURE 3. Four snapshots of the fluid pressure distribution (colour contours) and the tissue deformation at (a) $t / \mathcal{T}=9$, (b) $t / \mathcal{T}=9.25$ (c) $t / \mathcal{T}=9.5$, (d) $t / \mathcal{T}=9.75$. For each snapshot we show the system in its actual 1:1 aspect ratio (bottom) and with the radial dimensions stretched by a factor of 10 (middle and top). In the top panel the tissue displacements are exaggerated by a factor of 20 . The pressure is non-dimensionalised on the amplitude of the forcing pressure, $\widehat{P}$.

in Fig. 4(d) corresponds to $4.24 \mathrm{~cm} \mathrm{~s}^{-1}$, consistent with the range of pulsatile CSF velocities typically observed in MR scans.

Apart from the regions where transient vortex shedding occurs, the fluid pressure is approximately constant across any cross-sections through the SSS and the syrinx. The axial variation of the fluid pressure is illustrated in Figs. 5(a-d) which show four snapshots of the instantaneous (dashed lines) and cycle-averaged (solid lines) fluid pressure distribution along the FSI boundary between the SSS and the cord, and on the centreline of the syrinx (at $r=0)$. The instantaneous pressure distribution in the cranial part of the SSS is dominated by the time-harmonic pressure, $P_{\text {cranial }}(t)$, that we impose at $z=0$. Since $P_{\text {cranial }}(t)$ has zero mean, the cycle-average pressure in this region is close to zero. Conversely, the pressure in the caudal part of the SSS is strongly influenced by the viscous pressure drop across the narrow gap under the block. Since the width of this gap (and therefore the viscous flow resistance) varies considerably over the period of the oscillation (see Fig. 4(c)), nonlinear effects create a net pressure difference across the block, leading to an elevated cycle-average fluid pressure in the caudal part of the SSS the "valve mechanism" (Martin et al. 2010, Bertram 2010). The cycle-average pressures are shown in more detail in Fig. 5(e) where the arrows indicate the direction of the associated steady seepage flow that we expect to be generated by the transmural pressure difference across the porous syrinx cover.

For reference, the dashed-dotted line in this figure shows the cycle-average pressure distribution obtained in a simulation with an impermeable cord. As observed by Bertram 

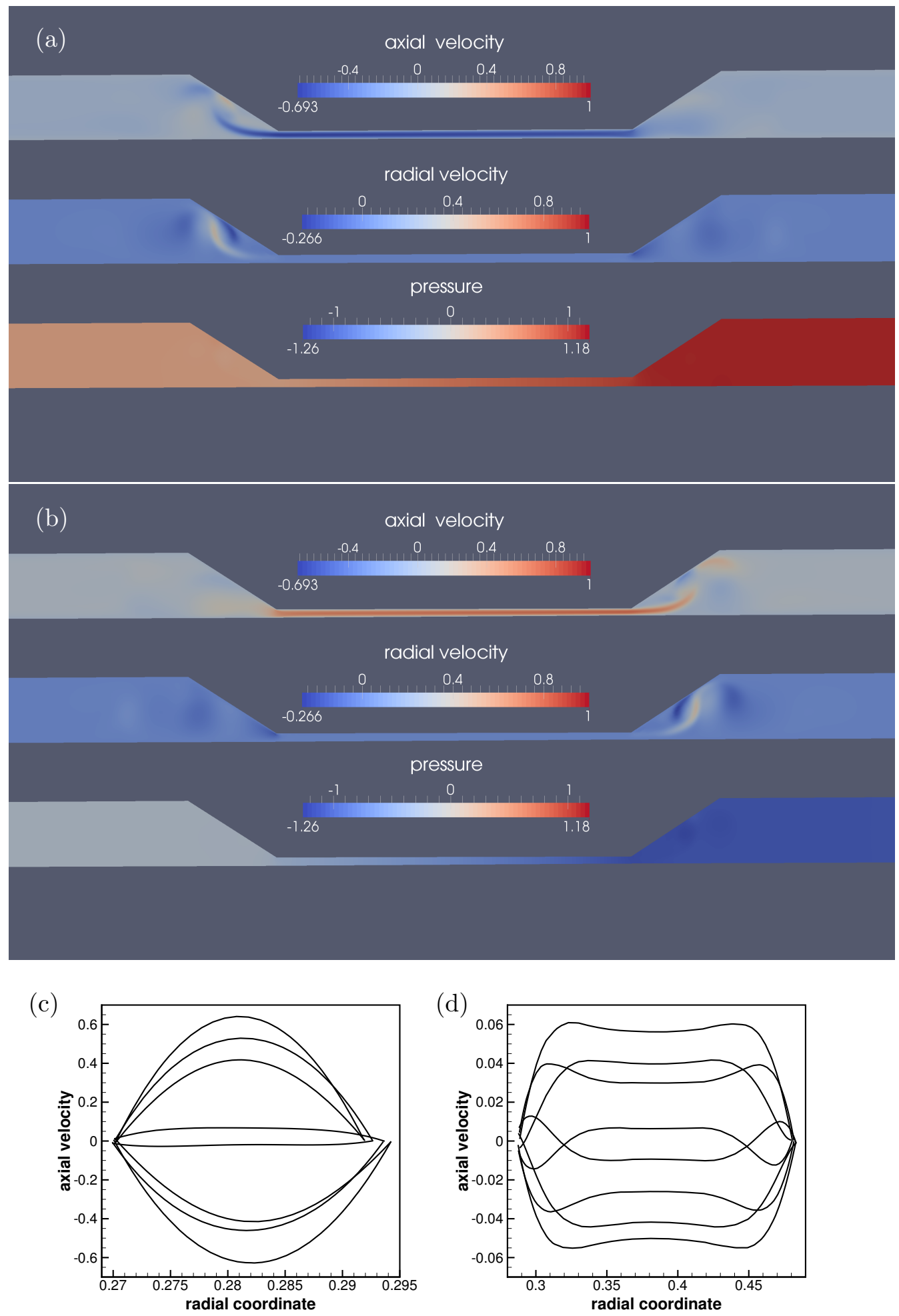

Figure 4. (a,b): Contour plots of the axial (top) and radial (middle) fluid velocities, and the fluid pressure (bottom) in the SSS in the vicinity of the block, plotted using the actual aspect ratio and the same orientation as in Fig. 1 . (a) $t / \mathcal{T}=9.25$, (b) $t / \mathcal{T}=9.75$. Bottom: profiles of the axial velocity in radial slices across the deforming SSS, $u_{z}(r, t)$, at (c) $z / \mathcal{D}=-7.5$ (in the gap between block and cord), (d) $z / \mathcal{D}=-3$ (in the cranial part of the SSS) at eight uniformly spaced instants over the forcing period, starting at $t / \mathcal{T}=9$. Velocities are scaled on the inertial velocity scale, $\mathcal{U}=\left(\widehat{\mathcal{P}} / \rho_{\text {fluid }}\right)^{1 / 2}$, and the pressure on the amplitude of the forcing pressure, $\widehat{\mathcal{P}}$. 


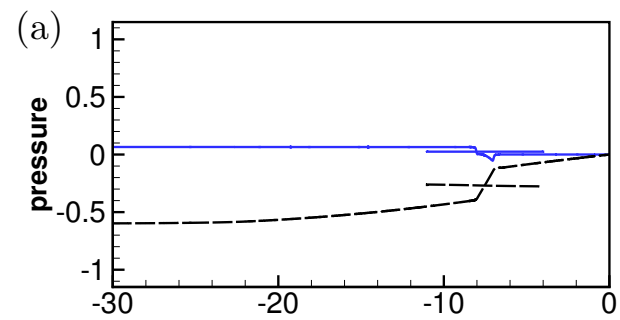

(c)

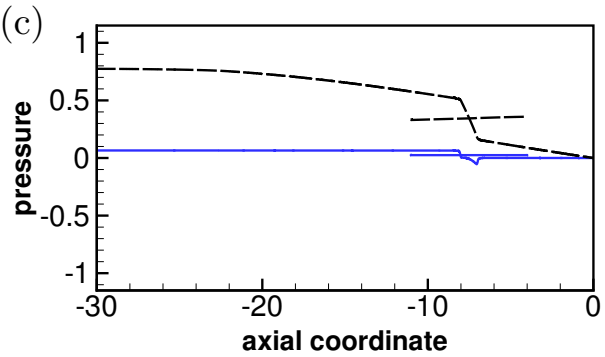

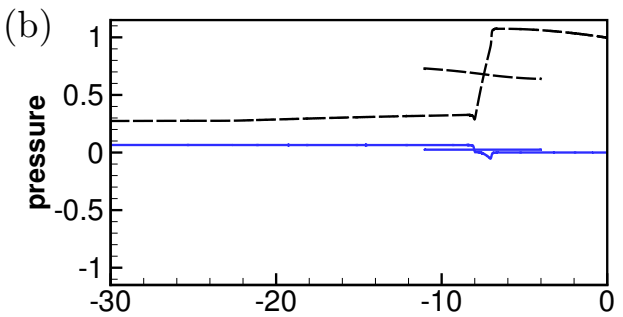

(d)

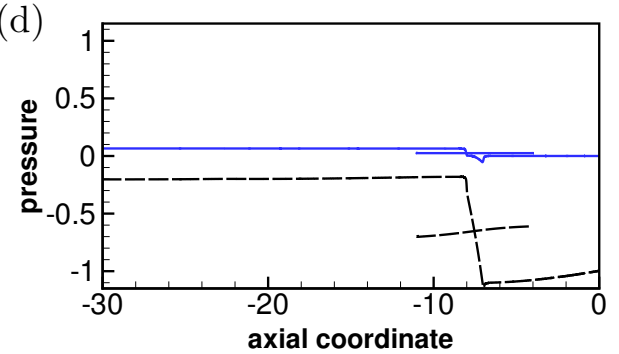

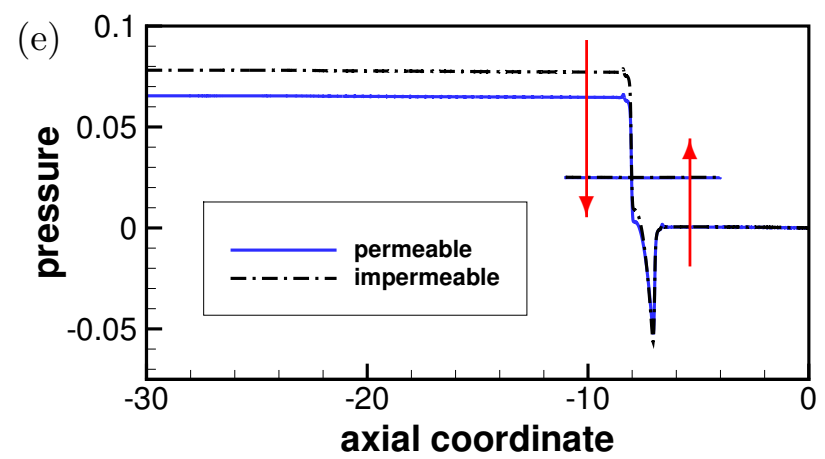

FiguRE 5. (a-d): Four snapshots of the instantaneous fluid pressure distribution in the SSS (along the FSI interface with the outer boundary of the cord; dashed line ranging over the entire length of the domain) and on the centreline of the syrinx (at $r=0$; dashed line ranging from $z / \mathcal{D}=-11$ to -4 ). (a) $t / \mathcal{T}=9$, (b) $t / \mathcal{T}=9.25$ (c) $t / \mathcal{T}=9.5$, (d) $t / \mathcal{T}=9.75$. The solid lines in Figs. (a)-(d) show the corresponding cycle-average pressures. These are shown more clearly in Fig. (e) where we also show (using a thick dash-dotted line) the pressure distributions in the corresponding impermeable system. All pressures are non-dimensionalised on the amplitude of forcing pressure, $\widehat{P}$, and the axial coordinate is scaled on the maximum diameter of the dura, $\mathcal{D}$.

(2010), in this case the pressure distribution is such that one would expect that, overall, fluid is driven from the SSS into the syrinx because the integral of the cycle-average transmural pressure, $\overline{\left.p\right|_{\text {SSS }}}-\overline{\left.p\right|_{\text {syrinx }}}$, over the length of the syrinx is positive. Here and elsewhere in this paper we denote the moving average of a quantity $f(t)$ as

$$
\bar{f}(t)=\frac{1}{\mathcal{T}} \int_{t}^{t+\mathcal{T}} f(\tau) d \tau,
$$



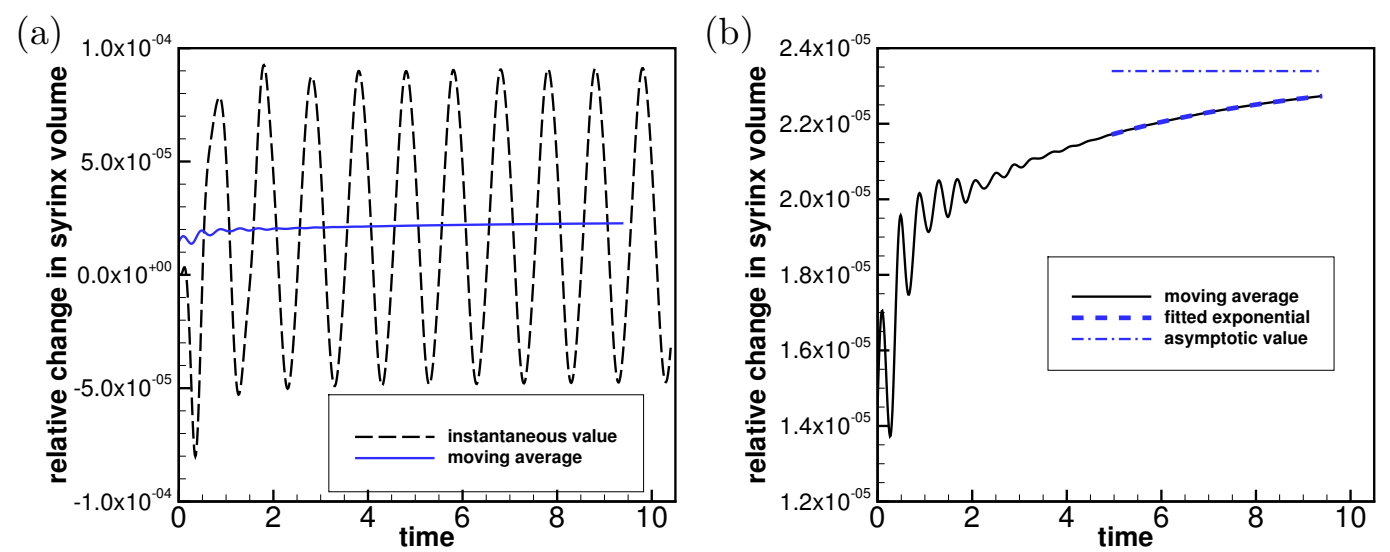

FIgURE 6. (a) Evolution of the relative change in syrinx volume, $\Delta V_{\text {syrinx }}(t) / V_{\text {syrinx }}(t=0)$ (black dashed line) and its cycle-average (blue solid line) for a permeability of $k=10^{-13} \mathrm{~m}^{2}$. (b) Plot of the cycle-average (black solid line), the fit to the exponential (4.2) (thick dashed line), and its asymptotic limit (thin dash-dotted line). Time is scaled on the period of the oscillation, $\mathcal{T}$.

and we omit the argument when we refer to the cycle-average over the ultimate timeperiodic steady state that is reached as $t \rightarrow \infty$, i.e. $\bar{f}=\lim _{t \rightarrow \infty} \bar{f}(t)$.

To assess if the permeability of the cord tissue does indeed result in a net increase in syrinx volume, Fig. 6(a) shows the evolution of the relative change in the syrinx volume, $\Delta V_{\text {syrinx }}(t) / V_{\text {syrinx }}(t=0)$. The syrinx volume fluctuates and, following the initial transients, settles into a periodic oscillation about a slowly evolving mean. To characterise the evolution of the latter, Fig. 6 (b) shows a plot of the moving average, $\overline{\Delta V_{\text {syrinx }}}(t)$, again normalised by the initial syrinx volume. We observe that, following the decay of the initial transients, the moving average tends to an asymptotic limit, $\Delta V_{\mathrm{syrinx}}^{[\infty]}$, the approach to which is well described by a damped exponential, i.e.

$$
\overline{\Delta V_{\text {syrinx }}}(t) \approx \Delta V_{\text {syrinx }}^{[\infty]}+A \exp (-\lambda t) \quad \text { as } t \rightarrow \infty .
$$

This is illustrated by the broken lines in Fig. 6(b) which show the damped exponential (4.2) and its asymptotic limit, respectively, using parameter values for $\Delta V_{\text {syrinx }}^{[\infty]}, A$ and $\lambda$ that were obtained by fitting (4.2) to the computational data for $t>5 \mathcal{T}$.

Fig. 7 shows that the general trends observed in Fig. 6 for the default permeability of $k=10^{-13} \mathrm{~m}^{2}$ are robust when the permeability is changed over two orders of magnitude. The main effect of an increase in permeability is an increase in (i) the amplitude of the fluctuations in the syrinx volume; (ii) the asymptotic limit of its moving average; (iii) the rate at which this asymptotic limit is approached. In all cases, the poroelastic fluid-structure interaction results in a net increase (albeit relatively small) in the syrinx volume, $\Delta V_{\text {syrinx }}^{[\infty]}>0$.

The fact that the cycle-average syrinx volume approaches a constant value, $\Delta V_{\text {syrinx }}^{[\infty]}$, implies that the net porous seepage flow into the syrinx (integrated over one period of the oscillation) must tend to zero as $t \rightarrow \infty$. The plots in Fig. 8 illustrate how this is achieved: Fig. $8(\mathrm{~b})$ shows the cycle-averaged traction, $-\overline{p_{\text {pore }}} \mathbf{n}$, that is exerted onto the 
(a)

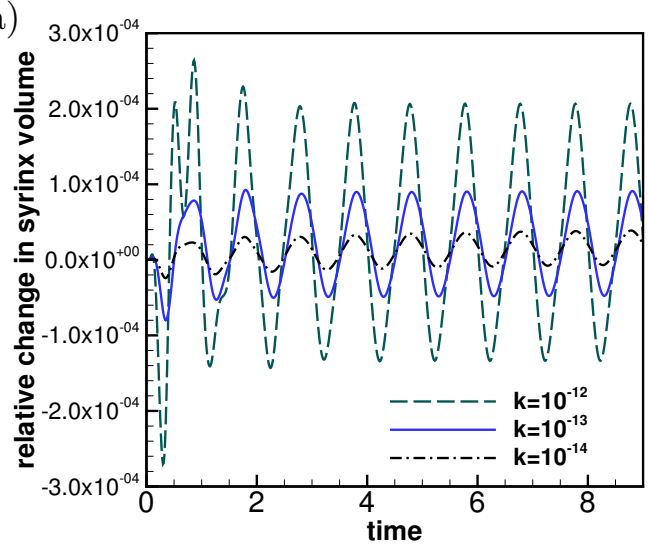

(b)

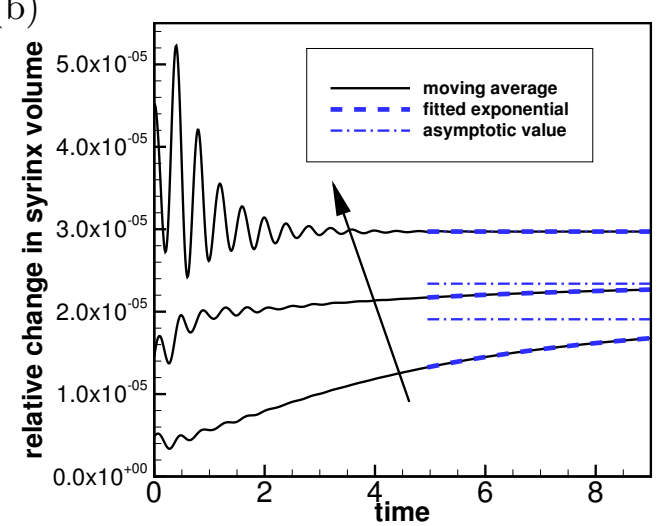

FiguRE 7. (a) Evolution of the relative change in syrinx volume, $\Delta V_{\text {syrinx }}(t) / V_{\text {syrinx }}(t=0)$ for three different values of the permeability. (b) Plot of the cycle-averages (black solid lines), the fits to the exponential (4.2) (thick dashed line), and their asymptotic limits (thin dash-dotted line). $k=10^{-14} \mathrm{~m}^{2}, 10^{-13} \mathrm{~m}^{2}, 10^{-12} \mathrm{~m}^{2}$ increasing in the direction of the arrow. Time is scaled on the period of the oscillation, $\mathcal{T}$.

pore fluid at the cord's FSI interfaces (see equation (2.13)), while Fig. 8(a) shows the normal component of the resulting cycle-average seepage flow, represented by the vector $(\overline{\mathbf{q}} \cdot \mathbf{n}) \mathbf{n}$. The cycle-average fluid pressure in the syrinx is approximately constant (see also Fig. 5), therefore the cycle-average transmural pressure, $\bar{p}_{\text {syrinx }}-\bar{p}_{\text {SSS }}$, that drives the seepage flow through the syrinx cover closely follows the variations of the fluid pressure in the SSS. This results in the generation of a mean flow in a closed loop, circulating as indicated by the oval in Fig. 8(a). This circular flow transports fluid from the cranial to the caudal end of the SSS where it seeps into the syrinx before re-emerging near the cranial end of the SSS. The reduction in the cycle-average pressure in the caudal part of the SSS (compared to the impermeable case), shown in Fig. 5(e), can therefore be seen to be a consequence of the need to maintain overall mass conservation. Since the seepage flow is relatively weak, the overall pressure distribution in the SSS (in particular, the elevated pressure in the caudal part of the SSS) remains very similar to that observed in the impermeable case. As a result, the "valve mechanism" remains active and generates an overall fluid pressure distribution that tends to drive fluid into [out of] the syrinx at its caudal [cranial] end. The initial imbalance between in- and outflow leads to a small increase in syrinx volume, but over sufficiently long times the fluid pressures adjust such that the net inflow into the syrinx exactly balances the net outflow. Fig. 5(e) shows that this is achieved predominantly by a reduction in the fluid pressure in the caudal part of the SSS (via small changes to the width of the gap under block and thus the associated flow resistance) rather than an increase in the fluid pressure in the (slightly inflated) syrinx.

Returning now to Fig. 8(a), we note that the majority of the seepage flow passes through the thin syrinx cover, while only a relatively small fraction of the overall flow leaves/enters the syrinx through its conical ends. The seepage flow has (integrable) singularities at the "corners" of the syrinx boundary (on the centreline and where the conical 
(a)

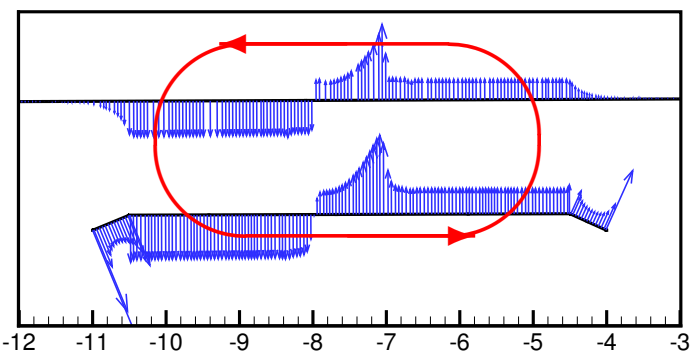

(b)

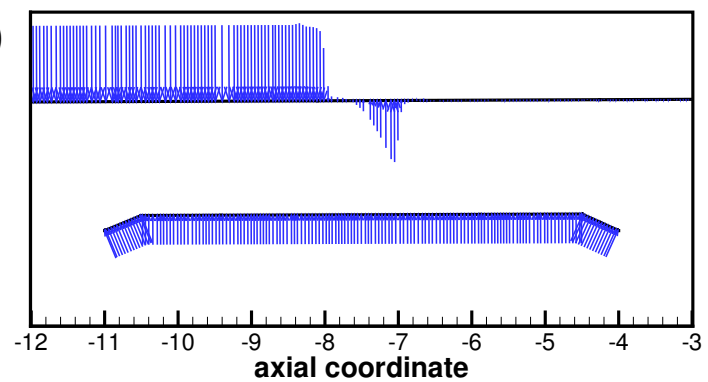

Figure 8. (a) Cycle-average seepage velocity, $(\overline{\mathbf{q}} \cdot \mathbf{n}) \mathbf{n}$, crossing the FSI interfaces between the cord and the SSS (top) and the syrinx (bottom). The FSI interfaces (black lines) have been separated vertically for clarity but are otherwise drawn using the actual aspect ratio. The closed loop indicates the direction of the circular seepage flow. (b) Cycle-average normal traction $-\bar{p}$ pore $\mathbf{n}$, acting onto the cord. All for $k=10^{-13} \mathrm{~m}^{2}$. The axial coordinate is scaled on the maximum diameter of the dura, $\mathcal{D}$.

ends meet the syrinx cover). These singularities are clearly artefacts of the overly simplistic syrinx geometry assumed in our model. We refer to $\$ 4.1 .2$ for a more detailed discussion of these features and an assessment of the sensitivity of our results to changes in the syrinx geometry. A detailed inspection of the seepage velocity field within the syrinx cover shows that the seepage flow is approximately unidirectional and directed radially inwards/outwards. The variation of the radial seepage velocity, $q_{r}$, and the pore pressure, $p_{\text {pore }}$, in a radial slice through the syrinx cover at $z=-7.5$ are illustrated in Fig. 9; these profiles are qualitatively similar to those in other cross-sections. The temporal fluctuations in both quantities are much larger than their cycle-averages, but they are several orders of magnitude smaller than the fluid velocities in the SSS and the syrinx. (Compare, e.g., the magnitudes of the seepage velocities, Fig. 9(a), to the fluid velocities in the SSS, Fig. 4 ; both are scaled on the inertial velocity scale, $\mathcal{U}=\left(\widehat{P} / \rho_{\text {fluid }}\right)^{1 / 2}$. $)$ The strong spatial variations in $q_{r}$ and, in particular, its deviation from a divergence-free profile, $q_{r} \sim 1 / r$, indicates that much of the seepage flow is generated by the dynamic compression/expansion of the solid matrix - as discussed in $\S 2$, the divergence of the solid velocity acts as a source term in the continuity equation for the seepage flow; see equation (2.8). The majority of the fluid that instantaneously enters the syrinx cover at the interface with the SSS is absorbed internally, with very little fluid emerging into the syrinx. In fact, over most of the period of the oscillation fluid tends to be ingested into (or 

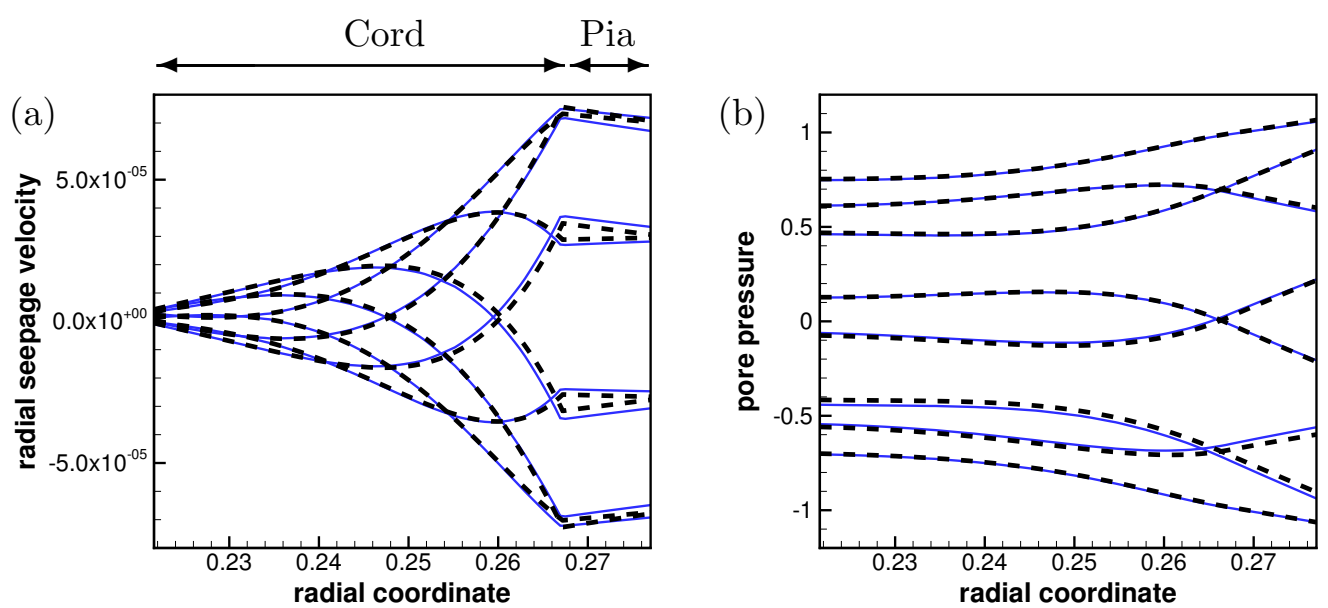

Figure 9. Profiles of (a) the radial component of the seepage velocity, $q_{r}(r, t)$, (b) the pore pressure, $p_{\text {pore }}(r, t)$, in a radial slice through the syrinx cover $($ at $z / \mathcal{D}=-7.5)$ at eight uniformly spaced instants over the forcing period, starting at $t / \mathcal{T}=9$. The blue solid line shows the results from the full FSI model; the black dashed lines show the predictions from the analytical model discussed in $\S 4.1$, where we approximated the forcing due to the fluid pressure and the axial strain by their first two Fourier components. The seepage velocity is scaled on the inertial velocity scale, $\mathcal{U}=\left(\widehat{\mathcal{P}} / \rho_{\text {fluid }}\right)^{1 / 2}$, the pore pressure on the amplitude of the forcing pressure, $\widehat{\mathcal{P}}$, and the radial coordinate on the maximum diameter of the dura, $\mathcal{D}$.

ejected from) the porous syrinx cover simultaneously at both FSI interfaces, indicating that the Darcy-like through flow that is driven by the instantaneous transmural pressure across the syrinx cover only makes a small contribution to the instantaneous seepage flow.

\subsection{A model for the seepage flows in the syrinx cover}

To elucidate the mechanism responsible for the structure of the seepage flows within the syrinx cover, we will now develop a simple analytical model that exploits the following observations: (i) over most of the syrinx cover (away from its cranial and caudal ends) the seepage velocity and the displacement of the solid matrix are dominated by their radial components; (ii) all quantities vary slowly with the axial coordinate, so that generally $\partial / \partial z \ll \partial / \partial r$; (iii) the traction exerted onto the poroelastic solid (and the embedded pore fluid) by the fluid in the SSS and the syrinx is dominated by the fluid pressure the temporal variation of which is well described by the first two Fourier components (comprising a constant part and a time-harmonic with period $\mathcal{T}$ ); (iv) the axial strain $\partial u_{z} / \partial z$ is approximately constant across the thickness of the syrinx cover and its temporal variation is again well described by the first two Fourier components. Combining these observations with the fact that the internal and external radii of the syrinx cover only vary slowly with $z$ suggests the use of a long-wavelength model in which each axial "slice" of the syrinx cover (treated as a thick-walled ring of inner and outer radius $a$ and $b$, respectively) is assumed to deform independently of its neighbours, and purely 
in response to the local traction acting on it. In the interest of brevity we present our derivation only for the case of a homogeneous syrinx cover, i.e. we do not distinguish between the pia and the cord (which have different constitutive properties; see $\$ 2.2$ ). The extension to the multi-layer case (for which we show results below) is straightforward. We assume that within a slice through the syrinx cover (i.e. at a fixed $z$-coordinate), the pore pressure and the radial components of the solid displacement and the seepage flow are given by

$$
\left\{p_{\text {pore }}(r, t), u_{r}(r, t), q_{r}(r, t)\right\}=\Re\left(\sum_{j}\left\{P_{\text {pore }}^{[j]}(r), U^{[j]}(r), Q^{[j]}(r)\right\} \exp \left(j 2 \pi \mathrm{i} \frac{t}{\mathcal{T}}\right)\right),
$$

and that the boundary conditions (2.12) and (2.13) can be approximated by

$$
\left.p_{\text {pore }}\right|_{r=a}=P_{\text {syrinx }}(t) \text { and }\left.\quad p_{\text {pore }}\right|_{r=b}=P_{\text {SSS }}(t)
$$

and

$$
-\left.\mathbf{n} \cdot(\boldsymbol{\sigma} \cdot \mathbf{n})\right|_{r=a}=P_{\text {syrinx }}(t) \text { and }-\left.\mathbf{n} \cdot(\boldsymbol{\sigma} \cdot \mathbf{n})\right|_{r=b}=P_{\mathrm{SSS}}(t) .
$$

We consider the system's response to imposed time-periodic variations in the fluid pressures and the axial strain,

$$
\left\{P_{\text {syrinx }}(t), P_{\text {SSS }}(t), \frac{\partial u_{z}}{\partial z}(r, t)\right\}=R e \sum_{j}\left\{\mathbb{P}_{\text {syrinx }}^{[j]}, \mathbb{P}_{\text {SSS }}^{[j]}, \mathbb{D}_{z}^{[j]}\right\} \exp \left(j 2 \pi \mathrm{i} \frac{t}{\mathcal{T}}\right)
$$

which are characterised by the Fourier coefficients $\mathbb{P}_{\text {syrinx }}^{[j]}, \mathbb{P}_{\mathrm{SSS}}^{[j]}, \mathbb{D}_{z}^{[j]}$, assumed to be given.

Since the poroelastic equations $(2.3)-(2.7)$ are linear, the different Fourier modes in (4.3) remain uncoupled. Upon inserting the ansatz (4.3) into equations (2.3)-(2.7) and neglecting any $z$-derivatives we therefore obtain one system of linear ODEs (for $U^{[j]}(r), Q^{[j]}(r), P_{\text {pore }}^{[j]}(r)$ ) for each Fourier mode $j$. While these systems are easily solved, further insight into the structure of the solution for $j>0$ can be obtained by observing that for the forcing frequencies considered here the inertial terms (on the right hand side of equations (2.3) and (2.4)) have little direct effect on the deformation of the syrinx cover (but see $\S 4.2$ for a more detailed discussion of the effects of solid inertia). Neglecting these terms allows us to combine equations (2.3)-(2.7) into a single equation for the solid displacement,

$$
\begin{aligned}
\frac{\mathrm{i} k \mathcal{T}}{2 \pi j \alpha^{2} \mu_{\text {fluid }}} \nabla \cdot \nabla \cdot & \frac{E_{\text {drained }}}{\left(1+\nu_{\text {drained }}\right)}\left(\frac{\nu_{\text {drained }}}{\left(1-2 \nu_{\text {drained }}\right)}\left(\nabla \cdot \mathbf{U}^{[j]}\right) \mathbf{I}+\frac{1}{2}\left(\nabla \mathbf{U}^{[j]}+\left(\mathbf{U}^{[j]}\right)^{T}\right)\right) \\
& +\nabla \cdot \mathbf{U}^{[j]}=0 \quad(\text { for } j>0),
\end{aligned}
$$

where $\mathbf{U}^{[j]}=U^{[j]}(r) \mathbf{e}_{r}+\mathbb{D}_{z}^{[j]} z \mathbf{e}_{z}$ - written in symbolic, vector-based notation because it keeps the equation in its most compact form. Equation (4.7) shows that the limit $k \rightarrow 0$ is singular since it causes the highest derivatives to vanish. For small but finite values of the permeability $k$ we therefore expect the existence of an interior region inside the syrinx cover within which the system's behaviour is well described by the equations for $k=0$, with boundary layers required to satisfy the boundary conditions (4.4) and (4.5). Setting $k=0$ in (4.7) shows that the solid displacement in the interior region must be divergence-free, $\nabla \cdot \mathbf{U}^{[j]}=0$, implying that

$$
U^{[j]}(r)=\frac{A}{r}-\frac{1}{2} \mathbb{D}_{z} r
$$

for some constant $A$. It turns out that, for a displacement field of this form, the divergence of the stress associated with the drained solid matrix vanishes, $\nabla \cdot \boldsymbol{\sigma}_{\text {elast }}^{[j]}=\mathbf{0}$, therefore 
equations (2.3) (with the inertial terms neglected) and (2.6) imply that the pore pressure is spatially constant, $\nabla p_{\text {pore }}^{[j]}=\mathbf{0}$. Substituting this into equation (2.4) then shows that the seepage flow vanishes so that in the interior of the syrinx cover we have

$$
Q^{[j]}(r)=0 \quad \text { and } \quad P_{\text {pore }}^{[j]}(r)=\text { const. }
$$

As expected, this solution does not allow us to satisfy all the boundary conditions and therefore requires the presence of boundary layers. These are indeed present in the full solution for the radial displacement which, in regions where the material properties are homogeneous, is given by

$$
U^{[j]}(r)=\underbrace{\frac{A}{r}-\frac{1}{2} \mathbb{D}_{z} r}_{\text {inner solution }}+\underbrace{B J_{1}\left(\mathrm{i}^{-1 / 2}\left(\frac{r}{\delta_{j}}\right)\right)+C K_{1}\left(\mathrm{i}^{-3 / 2}\left(\frac{r}{\delta_{j}}\right)\right)}_{\text {boundary layers }},
$$

where $J_{1}$ and $K_{1}$ are the Bessel function of the first kind and the modified Bessel function of the second kind (both of first order), respectively. In this expression the boundary layer thickness for the $j$-th Fourier mode is

$$
\delta_{j}=\left(\frac{k E_{\text {drained }}\left(1-\nu_{\text {drained }}\right) \mathcal{T}}{2 \pi j \alpha^{2} \mu_{\text {fluid }}\left(1+\nu_{\text {drained }}\right)\left(1-2 \nu_{\text {drained }}\right)}\right)^{1 / 2} .
$$

This agrees with the estimate of the boundary layer thickness in a time-periodically forced planar poroelastic layer (see, e.g., Detournay \& Cheng (1993)). The constants $A, B$ and $C$ in equation (4.10) follow from the boundary conditions at $r=a, b$. For the multi-layer case, where a solution of the form (4.10) describes the displacement field within each layer of the syrinx cover (i.e. within the pia and the cord itself) the imposition of the continuity of the radial displacement and stress, as well as the seepage flow and pore pressure at the interfaces between the two layers provides additional matching conditions. Similar expressions can be derived for $P_{\text {core }}^{[j]}$ and $Q^{[j]}$ by lengthy but straightforward algebra.

\subsubsection{Seepage flow in the syrinx cover}

To assess how well the approximate model manages to capture the key features of the seepage flow in the syrinx cover, we determined the Fourier coefficients $\mathbb{P}_{\text {syrinx }}^{[j]}, \mathbb{P}_{\text {SSS }}^{[j]}$ and $\mathbb{D}_{z}^{[j]}$ for the forcing that acts in the axial slice at $z=-7.5$ from the full FSI simulations. The dashed lines in Fig. 9 show the predictions for the seepage flow and the pore pressure obtained from the simplified analytical model with just two Fourier terms $(j=0,1)$. The predictions agree extremely well with the results from the fully coupled FSI simulation. Similar agreement was obtained in other cross-sections and for different values of the permeability. This indicates that our simplified model provides a good description of the poroelastic behaviour of the syrinx cover. We can therefore employ this model to study (at greatly reduced computational cost) the effect of variations in the tissue's permeability and its multi-layer structure. To illustrate the effect of the former, Figs. 10(a-c) show plots of the radial component of the seepage velocity, $q_{r}$, (left) and the pore pressure, $p_{\text {pore }}$, (right) for various values of the permeability, for the case of a homogeneous syrinx cover in which the drained Young's modulus of the pia was set to the same value as that of the cord. All other parameters (the Fourier coefficients $\mathbb{P}_{\text {syrinx }}^{[j]}, \mathbb{P}_{\mathrm{SSS}}^{[j]}$ and $\mathbb{D}_{z}^{[j]}$ and the dimension of the slice) were kept as in Fig. 9. For $k=10^{-13} \mathrm{~m}^{2}$ (Fig. 10(a)) the (nominal) boundary layer thickness is close to $20 \%$ of the thickness of the syrinx cover and as a result no pronounced boundary layers are visible in the various profiles; the combination of the driving pressure drop, $P_{\text {syrinx }}(t)-P_{\mathrm{SSS}}(t)$, and the kinematic 
redistribution of the pore fluid due to the compression/expansion of the solid matrix generates a seepage flow throughout the syrinx cover. As the permeability is reduced (to $k=10^{-14} \mathrm{~m}^{2}$ and $k=10^{-15} \mathrm{~m}^{2}$ in Figs. 10(b) and (c), respectively) the pore fluid becomes increasingly immobile. Since the pore fluid and the material that makes up the solid matrix are both assumed to be incompressible, the compound poroelastic medium becomes itself increasingly incompressible. The pore pressure in the interior becomes spatially constant and adopts a value that is, in general, inconsistent with the boundary conditions (4.4) and (4.5). The pore pressure therefore adjusts within the thin poroelastic boundary layers within which the pressure gradient becomes sufficiently large to generate significant seepage flows despite the tissue's small permeability.

Equation (4.11) shows that the thickness of the poroelastic boundary layers depends on the material's (drained) Young's modulus, $E_{\text {drained. }}$ In the full multi-layer model the stiffness of the pia is much greater than that of the cord, and the (nominal) boundary layer thickness in the pia exceeds its thickness, even for the smallest permeability considered here. As a result, the pore pressure and the seepage flow vary considerably throughout the pia, even in cases where the small permeability has virtually suppressed the seepage flow in the central region of the the cord (see Fig. 10(d) for $k=10^{-15} \mathrm{~m}^{2}$ ). Even though the pia is very thin, its stiffness is so much greater than that of the cord tissue that the pia contributes significantly to the overall stiffness of the syrinx cover. As a result, the amplitude of the syrinx displacements is reduced by a factor of $\approx 23$ compared to the case when the entire syrinx cover is assumed to be spatially homogeneous (with the constitutive properties of the cord tissue, as in Figs. 10(a-c)). The fact that the seepage flow within the syrinx is reduced by approximately the same factor (compare Figs. 10(c) and (d)) shows, yet again, that the seepage flow is dominated by the kinematic redistribution of the pore fluid due to the dynamic compression/expansion of the solid matrix.

We note that Figs. 10(a-d) contain a second set of (blue solid) lines. These were obtained numerically by time-integrating the full equations of poroelasticity (2.3)-(2.7) (using oomph-lib) in a finite-size, axially-uniform annular region of the same inner and outer radius as in the analytical model. The computations were performed using the forcing specified by equation (4.4) and (4.5). The annular region was allowed to expand freely in the radial direction at its axial ends where we suppressed any axial outflow and imposed the required value of $\partial u_{z} / \partial z$ by prescribing a suitable time-periodic axial displacement. The excellent agreement between the analytical and numerical results provides useful cross-validation between the two approaches. The direct numerical simulations also showed that for small permeabilities, it takes extremely long for the system to settle into its fully time-periodic state. For instance, the computations for $k=10^{-15} \mathrm{~m}^{2}$ had to be continued to 100 periods of the oscillation to achieve the level of agreement with the time-periodic analytical solution shown in Figs. 10(c,d). Such computations would have been extremely time-consuming if they had been performed with the fully-coupled FSI model.

\subsubsection{Implications for the change in syrinx volume}

The simplified analytical model also allows insight into the mechanism that is responsible for the change in syrinx volume - or, rather, it helps explain why the change is so small. For simplicity we only present the analysis for the case of a homogeneous syrinx cover; the results for the multi-layer case are algebraically significantly more complicated but the final outcome of the analysis is the same. We start by noting that only the steady components $(j=0)$ of all fields in equation (4.3) make a contribution to their cycle-averages - the other components have zero temporal mean by construction. Set- 
(a) $\geq$
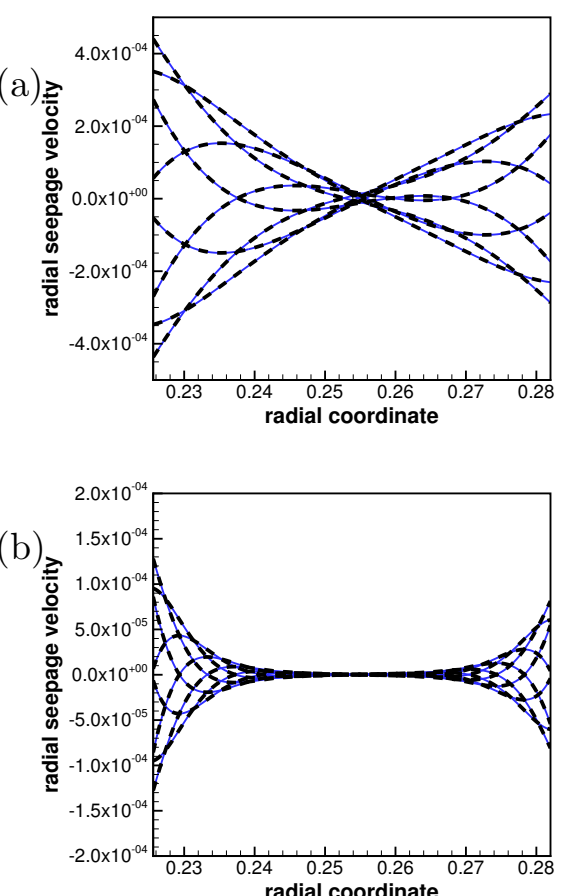

(c)
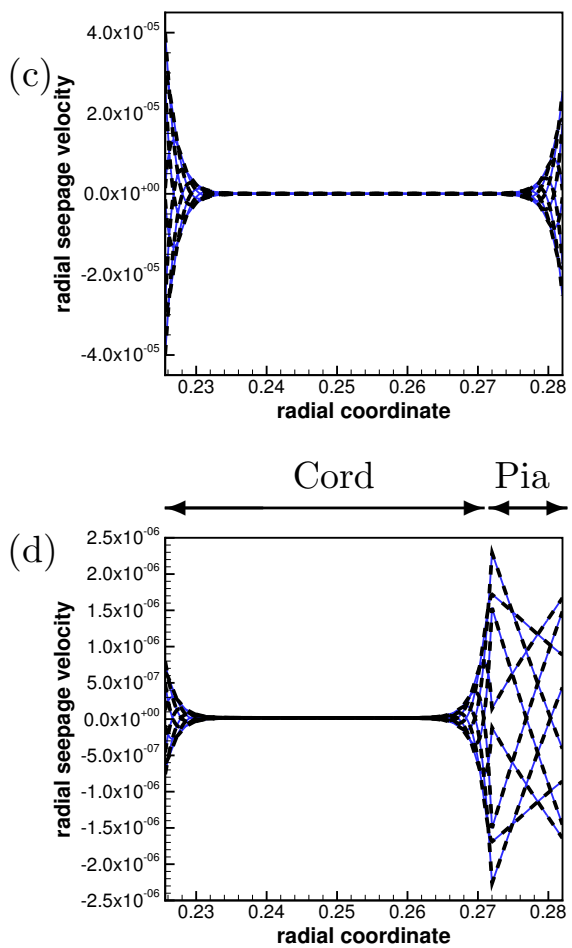
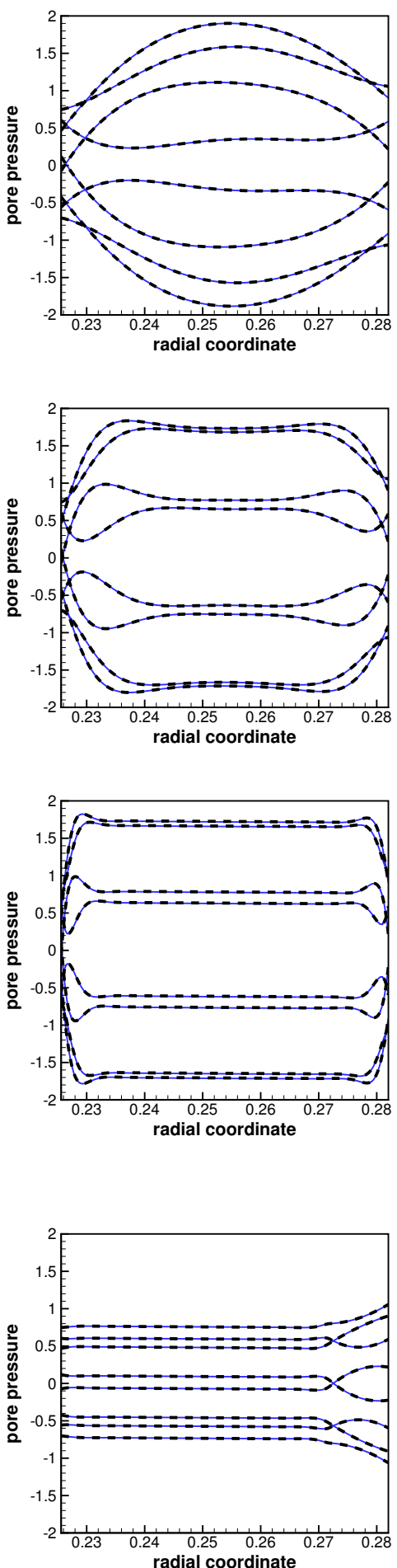

Figure 10. Profiles of the radial component of the seepage velocity, $q_{r}(r, t)$, (left), and the pore pressure, $p_{\text {pore }}(r, t)$, (right) in a radial slice through an axially uniform model of the syrinx cover at eight uniformly spaced instants over the forcing period. The blue solid line shows the results from the finite element-based solution of the poroelastic equations (2.3)-(2.7), time-integrated until a time-periodic steady state was established; the black dashed lines show the predictions for the time-periodic state from the analytical model discussed in $\S 4.1$. (a) $k=10^{-13} \mathrm{~m}^{2}$, (b) $k=10^{-14} \mathrm{~m}^{2},(\mathrm{c}, \mathrm{d}) k=10^{-15} \mathrm{~m}^{2}$. In (a-c) we made the syrinx cover uniform by setting $E_{\text {drained }}^{\text {[pia] }}=E_{\text {drained }}^{\text {[cord] }}$ in $(\mathrm{d})$ we employed the actual stiffnesses, resulting in a much stiffer pia. The seepage velocity is scaled on the inertial velocity scale, $\mathcal{U}=\left(\widehat{\mathcal{P}} / \rho_{\text {fluid }}\right)^{1 / 2}$, the pore pressure on the amplitude of the forcing pressure, $\widehat{\mathcal{P}}$, and the radial coordinate on the maximum diameter of the dura, $\mathcal{D}$. 
ting the time-derivative in equation (2.8) to zero shows that the steady seepage flow is divergence-free. Taking the divergence of the steady version of equation (2.4) therefore yields a second-order ODE for the pore pressure which is subject to the pressure boundary conditions $P_{\text {pore }}^{[0]}(r=a)=\mathbb{P}_{\text {syrinx }}^{[0]}$ and $P_{\text {pore }}^{[0]}(r=b)=\mathbb{P}_{\text {SSS }}^{[0]}$. The solution is given by

$$
P^{[0]}(r ; z)=\mathbb{P}_{\mathrm{SSS}}^{[0]}(z)-\left(\mathbb{P}_{\text {syrinx }}^{[0]}(z)-\mathbb{P}_{\mathrm{SSS}}^{[0]}(z)\right) \frac{\ln (r / a(z))}{\ln (b(z) / a(z))}
$$

Substituting this back into (2.4) shows that the steady part of the seepage velocity in a radial slice at an axial coordinate $z$ is given by the Darcy profile

$$
Q^{[0]}(r ; z)=\frac{k\left(\mathbb{P}_{\text {syrinx }}^{[0]}(z)-\mathbb{P}_{\text {SSS }}^{[0]}(z)\right)}{\mu_{\text {fluid }} \ln (b(z) / a(z))} \frac{1}{r}
$$

which depends linearly on the local transmural pressure $\mathbb{P}_{\text {syrinx }}^{[0]}(z)-\mathbb{P}_{\text {SSS }}^{[0]}(z)$ across the syrinx cover. Inserting (4.12) and (2.7) into (2.6) then yields the steady component of the stress tensor in the cord tissue which we substitute into the steady version of equation (2.3). The radial component of this equation provides a second-order ODE for the steady component of the radial displacement which is subject to the steady part of the two traction boundary conditions (4.5). Solving these equations yields

$$
\begin{aligned}
& U^{[0]}(r ; z)=\frac{1+\nu_{\text {drained }}}{E_{\text {drained }}\left(b^{2}(z)-a^{2}(z)\right)}(\underbrace{\left(\mathbb{P}_{\text {syrinx }}^{[0]}(z)-\mathbb{P}_{\mathrm{SSS}}^{[0]}(z)\right) \frac{a^{2}(z) b^{2}(z)}{r}}_{\mathrm{I}} \\
& +\underbrace{\left(a^{2}(z) \mathbb{P}_{\text {syrinx }}^{[0]}(z)-b^{2}(z) \mathbb{P}_{\text {SSS }}^{[0]}(z)\right)\left(1-2 \nu_{\text {drained }}\right) r}_{\text {II }})-\underbrace{\nu_{\text {drained }}^{\mathbb{D}_{z}^{[0]}(z) r}}_{\text {III }} \\
& +\frac{\alpha\left(1+\nu_{\text {drained }}\right)\left(1-2 \nu_{\text {drained }}\right)}{2 E_{\text {drained }}\left(1-\nu_{\text {drained }}\right)}\left\{\left(\frac{\left[\left(1-\nu_{\text {drained }}\right)-\ln (r / a(z))\right]\left(\mathbb{P}_{\text {syrinx }}^{[0]}(z)-\mathbb{P}_{\text {SSS }}^{[0]}(z)\right)}{\ln (b(z) / a(z))}\right.\right. \\
& \left.+\frac{\left(\mathbb{P}_{\text {syrinx }}^{[0]}(z)-\mathbb{P}_{S S S}^{[0]}(z)\right) b^{2}(z)-\nu_{\text {drained }}\left(\mathbb{P}_{\text {SSS }}^{[0]}(z) b^{2}(z)+\mathbb{P}_{\text {syrinx }}^{[0]}(z) a^{2}(z)\right)-\left(1-2 \nu_{\text {drained }}\right) \mathbb{P}_{\text {syrinx }}^{[0]}(z) a^{2}(z)}{b^{2}(z)-a^{2}(z)}\right) r \\
& \left.-\frac{a^{2}(z) b^{2}(z)\left(\mathbb{P}_{\text {syrinx }}^{[0]}(z)-\mathbb{P}_{\mathrm{SSS}}^{[0]}(z)\right)}{b^{2}(z)-a^{2}(z)} \frac{1}{r}\right\}
\end{aligned}
$$

Here term I represents the divergence-free expansion of the syrinx cover in response to the local transmural pressure, $\mathbb{P}_{\text {syrinx }}^{[0]}(z)-\mathbb{P}_{\text {SSS }}^{[0]}(z)$; term II represents the pressureinduced compression of the syrinx cover (note that this term vanishes if the drained solid matrix is incompressible, $\left.\nu_{\text {drained }}=1 / 2\right)$; term III arises from the fact that the axial compression of the syrinx cover (represented by $\mathbb{D}_{z}^{[0]}$ ) changes its thickness in proportion to Poisson's ratio. The remaining terms are pre-multiplied by the Biot-Willis parameter $\alpha$, indicating that they arise through the drag that the pore fluid exerts onto the solid matrix; interestingly, this effect again vanishes if the solid matrix is incompressible, i.e. if $\nu_{\text {drained }}=1 / 2$.

In the full FSI model, the inner and outer boundaries of the syrinx cover are only 
slightly tapered in the axial direction, therefore the radii $a(z)$ and $b(z)$ are approximately constant and close to their average values, $a_{0}$ and $b_{0}$, say. Assuming that the flow into/out of the syrinx is dominated by the flow across the syrinx cover (located between $z=z_{\text {lower }}$ and $z_{\text {upper }}$, say), the condition that the cycle-average in/outflow over the surface of the syrinx, $\partial V_{\text {syrinx }}$,

$$
\begin{aligned}
\oint_{\partial V_{\mathrm{syrinx}}} \mathbf{q}^{[0]} \cdot \mathbf{n} d S & =2 \pi \int_{z_{\text {lower }}}^{z_{\text {upper }}} Q^{[0]}(a(z) ; z) a(z) d z \\
& \approx \frac{2 \pi k}{\mu_{\text {fluid }} \ln \left(b_{0} / a_{0}\right)} \int_{z_{\text {lower }}}^{z_{\text {upper }}}\left(\mathbb{P}_{\text {syrinx }}^{[0]}(z)-\mathbb{P}_{\mathrm{SSS}}^{[0]}(z)\right) d z
\end{aligned}
$$

is zero therefore requires that

$$
\int_{z_{\text {lower }}}^{z_{\text {upper }}}\left(\mathbb{P}_{\text {syrinx }}^{[0]}(z)-\mathbb{P}_{\text {SSS }}^{[0]}(z)\right) d z=0 .
$$

We now exploit that the typical thickness of the syrinx cover $h_{\text {cover }}=b_{0}-a_{0}$ is small. Evaluating equation (4.14) at $r=a(z) \approx a_{0}$ and expanding the result in $h_{\text {cover }} / a_{0} \ll 1$ allows us to approximate the cycle-average radial displacement at the inner boundary of the syrinx cover by

$$
U^{[0]}(a(z) ; z) \approx\left(\mathbb{P}_{\text {syrinx }}^{[0]}(z)-\mathbb{P}_{\text {SSS }}^{[0]}(z)\right) \frac{a_{0}\left(1-\nu_{\text {drained }}^{2}\right)}{\left(h_{\text {cover }} / a_{0}\right) E_{\text {drained }}} .
$$

Unlike the full expression, equation (4.14), the approximate version (4.17) is linear in the the local transmural pressure, $\mathbb{P}_{\text {syrinx }}^{[0]}(z)-\mathbb{P}_{\text {SSS }}^{[0]}(z)$, across the syrinx cover. This implies that the change in syrinx volume induced by the radial deformation of the syrinx cover,

$$
\begin{aligned}
\Delta V_{\text {syrinx }}^{[\infty]} & =\oint_{\partial V_{\text {syrinx }}} \mathbf{u}^{[0]} \cdot \mathbf{n} d S=2 \pi \int_{z_{\text {lower }}}^{z_{\text {upper }}} U^{[0]}(r=a(z) ; z) a(z) d z \\
& \approx \frac{2 \pi a_{0}^{2}\left(1-\nu_{\text {drained }}^{2}\right)}{\left(h_{\text {cover }} / a_{0}\right) E_{\text {drained }}} \int_{z_{\text {lower }}}^{z_{\text {upper }}}\left(\mathbb{P}_{\text {syrinx }}^{[0]}(z)-\mathbb{P}_{\mathrm{SSS}}^{[0]}(z)\right) d z
\end{aligned}
$$

vanishes because mass conservation requires the integral in the final term to be zero; see (4.16).

Given that our model provides a good description of the behaviour of the syrinx cover, the small change in syrinx volume that is observed in the full FSI simulations must therefore arise predominantly through secondary features that are not captured by this model, such as the flow through and the deformation of the (conical) ends of the syrinx. To show that these do indeed have a (relatively large) effect on the (small) change in syrinx volume, we performed an additional FSI simulation using a different syrinx geometry. Rather than terminating the syrinx with conical ends (as in Bertram's (2010) original model), we used ellipsoidal ends which merged smoothly with the unchanged syrinx cover. Figs. 11(a,b) show that this has very little effect on the overall pressure distribution and the seepage flow along the syrinx cover. The main effect of the change in the geometry is the disappearance of the singularities in the seepage flow that developed at the sharp corners in the conical geometry (cf. Fig. 8).

Figs. 11(d-f) compare the cycle-average deformation of the syrinx for the two geometries and shows that the deformation of the syrinx cover for the two cases is graphically indistinguishable. In fact, even the deformation of the syrinx ends is practically identical in the sense that for both geometries it is dominated by their axial displacements. It is difficult to identify the mechanism via which the change in the syrinx geometry leads to the further increase in the syrinx volume that is evident in the time trace in 
Fig. 11(c). This is because even though the change in the syrinx geometry increases the relative change in syrinx volume by approximately $25 \%$, the net change in syrinx volume of $\mathcal{O}\left(10^{-3} \%\right)$ is far too small to be observable in the deformation shown in Figs. 11(d-f), despite the fact that the displacements are exaggerated by a factor of 40 in these plots.

To assess the robustness of our findings we also studied the effect of variations in the waveform of the imposed pressure at the cranial end, $P_{\text {cranial }}(t)$, by replacing the sinusoidal signal (2.16) with a waveform that more closely resembles actual cardiac-induced pressure fluctuations. While this forcing generated higher-frequency components in the system's response (results not shown in the interest of brevity), the overall behaviour (in terms of the tissue deformation, CSF flow and the character of the seepage flows) remained qualitatively unchanged. In particular, the forcing still generated only a very small increase in the cycle average syrinx volume - even smaller, in fact, than that generated by the sinusoidal forcing (2.16)).

Finally, we considered the effect of variations in the initial syrinx volume which we illustrate in Fig. 12(a). The figure shows time traces of the relative change in syrinx volume, created by the sinusoidal forcing (2.16), for four different syrinx shapes. These were generated by displacing the conical syrinx ends symmetrically in the axial direction, as illustrated in Fig. 12(b) which shows plots of the syrinx shapes (in the $(r, z)$ plane) using a 1:1 aspect ratio. The system's overall behaviour (again assessed in terms of the tissue deformation, CSF flow and the character of the seepage flows) was again found to be qualitatively similar in all cases and the change in the cycle average syrinx volume was always very small. A reduction/increase in the length of the syrinx can be seen to lead to a systematic reduction/increase in the relative change in syrinx volumes, so that for sufficiently short syrinxes the volume change becomes negative. This implies that the presence of seepage flow does not always lead to an inflation of the syrinx. Because the actual change in syrinx volume remained very small, we were again unable identify a simple mechanism that would explain how the volume change depends on the length of the syrinx - the differences between the various cases are too small to be observable, and there may, of course, not even be a single mechanism that is responsible for the observed behaviour. Similar behaviour is found when the position of the syrinx is shifted relative to the block; see Bertram \& Heil (2016).

\subsection{The effect of solid inertia and the slip boundary conditions}

For the forcing frequencies and permeabilities considered here, inertial effects do not have a strong effect on the deformation of and the seepage flow within the syrinx cover: the results obtained from our analytical model (which excluded the terms on the right-handside of equations (2.3) and (2.4)) agree perfectly with those from corresponding direct numerical simulations in which inertia was included. This is because the deformation of the syrinx cover is dominated by its (small) radial displacements. However, inertia does have a noticeable effect on the system's overall behaviour because the cord tissue undergoes relatively large time-periodic axial displacements (and hence accelerations). These do affect the fluctuations in syrinx volume but appear to have little effect on the its ultimate cycle-average; see Fig. 13(a). We also investigated the effect of variations in the porosity $\phi$ which, within our simple model for the behaviour of the poroelastic tissues, only features in the inertial terms for the pore fluid in equation (2.4). Repeating the simulations with a value of $\phi=0.03$ yielded results that were graphically indistinguishable from those obtained with the default value of $\phi=0.3$ - another indication that the seepage flow is far too weak to have a significant effect on the system's overall behaviour.

Fig. 13(b) demonstrates that the slip of the fluid along the porous FSI interfaces has 

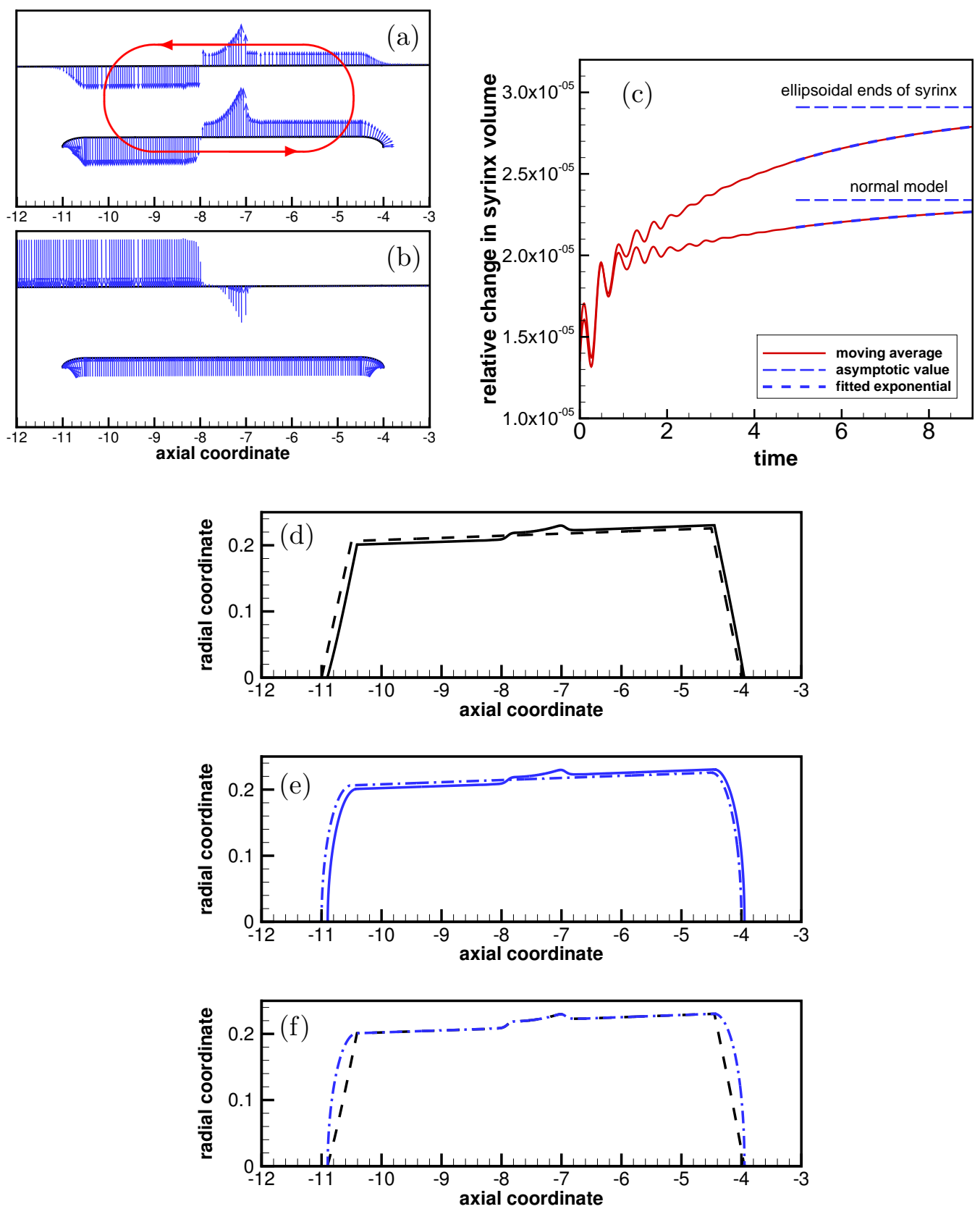

Figure 11. Plots illustrating the effect of changing the geometry of the syrinx. (a) and (b) show the cycle-average seepage velocity and normal traction acting onto the cord (as in Fig. 8; see caption to that figure for details) when the conical ends of the syrinx in the original model are changed to ellipsoids. (c) Cycle-average of the relative change in syrinx volume, $\overline{\Delta V_{\text {syrinx }}}(t) / V_{\text {syrinx }}(t=0)$ (red solid line), the fit to the exponential (4.2) (thick dashed line), and its asymptotic limit (thin dash-dotted line) for the two syrinx geometries. Time is scaled on the period of the oscillation, $\mathcal{T}$. (d-f): Comparison of the cycle-average syrinx deformation for the (d) original and (e) ellipsoidal syrinx shape. Broken lines: undeformed shape; solid lines: deformed shape. (f) Direct comparison between the deformed cycle-average syrinx shapes for the two geometries. All displacements are exaggerated by a factor of 40 and lengths are scaled on the maximum diameter of the dura, $\mathcal{D}$. 
(a)

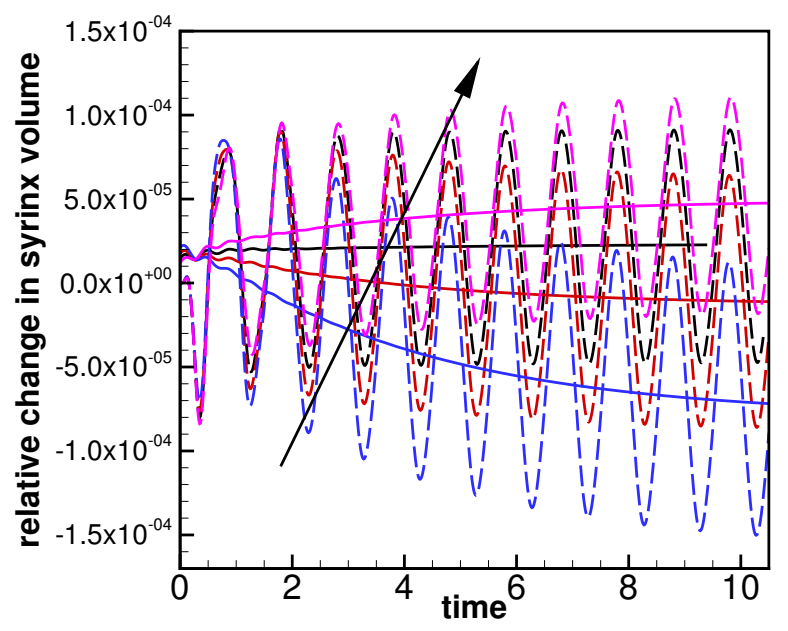

(b)

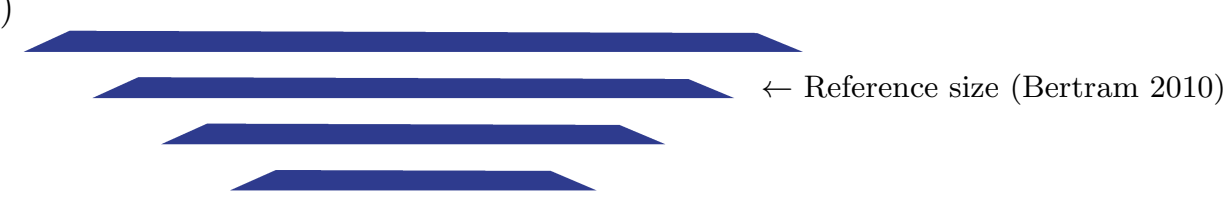

Figure 12. (a) Relative change in syrinx volume (instantaneous and cycle-averaged, as in Fig. 6) for $k=10^{-13} \mathrm{~m}^{2}$ for syrinxes of different length, increasing in the direction of the arrow. (b) Plot of the four syrinxes (in the $(r, z)$ plane) using a 1:1 aspect ratio.

little effect on the result. The slip coefficient $\gamma$ in equation (2.15) is proportional to the fluid's kinematic viscosity, $\gamma=\alpha_{\mathrm{BJS}} \mu_{\text {fluid }}$, where $\alpha_{\mathrm{BJS}}$ is an $\mathcal{O}(1)$ constant; see Beavers \& Joseph (1967). Equation (2.15) shows that slip effects are most likely to play a significant role for large permeabilities. We therefore repeated the simulation for $k=10^{-12} \mathrm{~m}^{2}$ (the largest permeability considered in this study) with a non-zero value of the inverse slip coefficient, $\gamma^{-1}=\mu_{\text {fluid }}^{-1}$ (the value for $\alpha_{\mathrm{BJS}}=1$ ). Fig. 13(b) shows that this has no visible effect on the results.

\section{Summary and Discussion}

The results from the fully-coupled FSI simulations and from our simple analytical model provide a clear picture of the mechanism by which poroelastic fluid-structure interaction controls the mass transfer between the SSS and the syrinx. For the relatively small permeabilities encountered in the spinal cord (see below for a more detailed discussion of the constitutive models and their parameters), the system's overall behaviour is dominated by the interaction between the elastic deformation of the various tissues and the fluid flow in the SSS and the syrinx. This interaction remains very similar to that observed in Bertram's (2010) study of the corresponding impermeable system. In particular, nonlinear effects arising through the time-varying flow resistance across the narrow gap under the block create a cycle-average difference in fluid pressure between 

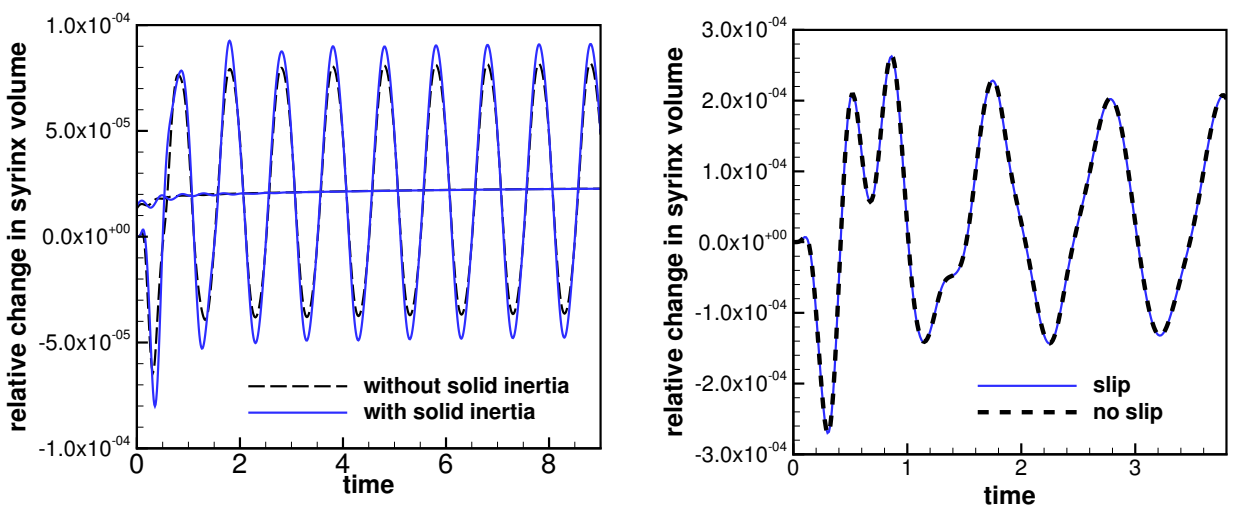

FiguRE 13. Relative change in syrinx volume (instantaneous and cycle-averaged, as in Fig. 6) for $k=10^{-13} \mathrm{~m}^{2}$ with (solid) and without (dashed) the inertial terms in equations (2.3) and (2.4). (b) Relative change in syrinx volume for $k=10^{-12} \mathrm{~m}^{2}$, allowing $\left(\gamma^{-1}=\mu_{\text {fluid }}^{-1}\right.$; solid) and not allowing $\left(\gamma^{-1}=0\right.$; dashed) for tangential slip at the FSI interfaces.

the cranial and caudal part of the SSS - the "valve mechanism". In the impermeable system, the resulting transmural pressure across the syrinx cover is such that it has a tendency to drive fluid from the SSS into the syrinx. When the permeability of the tissue is accounted for, any initial imbalance in the cycle-average seepage flow into/out of the syrinx leads to a change in syrinx volume and an adjustment of the various fluid pressures until a new cycle-average steady-state is established. Computations show that the adjustment in the fluid pressures is achieved primarily by a small reduction in fluid pressure in the caudal part of the SSS, with the pressure in the syrinx and in the cranial part of the SSS remaining virtually unchanged compared to the impermeable case. The direction of the transmural pressure differences that drive fluid across the porous syrinx cover remains unchanged too and in the poroelastic system they generate a cycle-average circular seepage flow which transports fluid into/out of the syrinx in its caudal/cranial part. Once the system has reached its cycle-average steady state, there is no net mass transfer into the syrinx - the inflow exactly balances the outflow. We developed a simple analytical model which shows that for elongated syrinx geometries in which most of the seepage flow into/out of the syrinx passes through a long, thin syrinx cover, the requirement of zero cycle-average in/outflow implies that the change in the cycle-average syrinx volume is close to zero. This is consistent with the results from the full FSI model which showed that the change in syrinx volume is indeed very small. We note that the analysis presented in $\S 4.1 .2$ relied on the fact that for sufficiently thin syrinx covers, both the radial seepage flux and the radial displacement of the syrinx cover depend linearly on the local transmural pressure difference across the syrinx cover. For thicker syrinx covers, all terms in equation (4.14) (including those that, like term II, do not depend on the transmural pressure difference alone) will affect the expansion (or compression) of the syrinx.

The steady circular seepage flow that develops after the decay of the initial transients results in a continuous mass transfer between the syrinx and the SSS. However, its mag- 
nitude is generally much smaller than that of the time-periodic fluctuations which are represented by the higher Fourier modes $(j>0)$ in the expansion (4.3). The unsteady flow is driven primarily by the kinematic redistribution of the fluid within the periodically expanding and contracting solid matrix. For sufficiently small permeabilities the oscillatory seepage flows and the pore pressures develop pronounced boundary layers at the FSI interfaces and at the material interfaces between the different tissue layers, e.g. between the pia and the cord. The thickness of the boundary layers at the FSI interfaces controls how far the oscillatory seepage flows penetrate the solid matrix.

The overall behaviour was found to be robust to changes in the waveform of the imposed pressure fluctuations, $P_{\text {cranial }}(t)$, and to changes in the shape and the size of the syrinx: while the precise value of the change in the cycle average syrinx volume was found to depend sensitively on these parameters (and, for sufficiently short syrinxes can even become negative), its value was always very small - possibly too small to regard it as a sufficiently strong stimulus for a subsequent remodelling of the cord tissue which would lead to a long-term growth of the syrinx. While, over sufficiently long timescales even a small stimulus may initiate/drive a remodelling process, our model neither permits us to explore this nor to pass judgement on the likelihood of other mechanisms (possibly non-mechanical in nature, such as inflammation) being at work. There is a considerable body of literature on mechanically-induced remodelling in the cardiovascular system (see, e.g., Humphreys (2008) for a review of arterial remodelling), but we are not aware of any comparable studies in the context of syringomyelia.

The model used in this study incorporates a fair amount of anatomical detail such as the multi-layer-structure of the spinal cord. However, in an attempt to facilitate the identification of the dominant mechanisms that control the mass transfer from the SSS to the syrinx, we deliberately applied many drastic simplifications: in vivo, neither the geometry nor the flow are perfectly axisymmetric; the SSS is traversed by nerve bundles and connective tissue filaments known as trabeculae; biological tissue is not perfectly poroelastic and its material properties are anisotropic and inhomogeneous; the dura is surrounded by other tissue which we omitted from our model (though we note that Bertram (2010) deliberately set the thickness of the dura to a value that is somewhat larger than in vivo in an attempt to account for the stiffness of the backing); etc.

Recent improvements in MRI-based imaging techniques have made it possible to visualise not only the geometry of the SSS but also the time-resolved 3D flow of CSF within it; see, e.g., Bunck et al. (2011), Yiallourou et al. (2015), Heidari Pahlavian et al. (2014), Heidari Pahlavian et al. (2015). These visualisations show that, as expected, deviations from an axisymmetric geometry tend to induce secondary non-axisymmetric flow features such as the formation of jets near localised constrictions, and recirculation regions downstream of fine-scale anatomical features such as nerve roots or denticulate ligaments. However, these complex localised flow features appear to have little effect on the average CSF flow (Stockman 2006), and more importantly, on the CSF pressure distribution along the SSS (Heidari Pahlavian et al. 2014) which we showed to be the key feature that controls the seepage flow from the SSS to the syrinx. Note, however, that simulations by Gupta et al. (2008) and Gupta et al. (2010) showed that in the cranial subarachnoid space the presence of the trabecular network has a significant effect on the CSF flow.

Our model assumed the system to be forced exclusively by the cranial pressure fluctuation, $P_{\text {cranial }}(t)$, which we assumed to be correlated with the heart beat. CSF flow can also be affected by respiratory manoeuvres, with sneezing and coughing providing particularly powerful, transient pressure perturbations; see, e.g., Elliott et al. (2013). While steady-state, quiet breathing generates pressure variations of much smaller amplitude, it may still have an effect on the CSF flows (see, e.g., Dreha-Kulaczewski et al. 2015). 
Regarding the constitutive modelling we note that, even though the spinal cord is viscoelastic (see, e.g., Chang \& Hung (1988)), for the forcing frequencies considered in the current study, viscoelastic effects only play a minor role. Furthermore, even though we did not include viscoelastic effects into the constitutive equation for the drained solid matrix, the dissipation associated with the seepage flows introduces viscous losses into the solid model; see, e.g., Elliott (2012) for a study of the effects of poroelasticity on the propagation of pressure waves along the SSS. Estimates for the elastic constitutive parameters such as the tissues' Young's moduli vary considerably and most tissues display noticeable anisotropy. We refer to Elliott et al. (2013) for a compilation of data from a variety of sources; in the current study we used the same (isotropic) values that were used by Bertram (2010) to facilitate comparisons between the two studies. Similar comments apply to parameter values for the tissue permeability where estimates span several orders of magnitude, ranging from $\mathcal{O}\left(10^{-14} \mathrm{~m}^{2}\right)$ for white matter to $\mathcal{O}\left(10^{-16} \mathrm{~m}^{2}\right)$ for gray matter (see, e.g., Smillie et al. 2005), with the permeability of the cord greater in the longitudinal than the transverse direction (Rossi et al. 2008). While it is relatively straightforward to include such details in a computational model, the determination of the appropriate patient-specific parameters is difficult. As a result, even in studies that are based on anatomically realistic geometries, "parameters are usually obtained experimentally from animal cadavers", to quote from Støverud et al. (2015) who assessed the sensitivity of a poroelastic model of the spinal cord (with anatomically correct geometry determined from MRI scans) to variations in the constitutive parameters.

While our comparatively simple model clearly cannot (and is not intended to) capture all of these details, we believe that its relative simplicity helps to elucidate the fundamental mechanisms by which poroelastic fluid-structure interaction controls the mass transfer between the SSS and the syrinx.

\section{Acknowledgements}

We acknowledge support from the Chiari and Syringomyelia Foundation (grant to CDB). Matthew J. Russell contributed to the implementation of the poroelastic FSI capabilities in oomph-lib. Draga Pihler-Puzovic provided many helpful comments on an earlier draft of this manuscript.

\section{REFERENCES}

Amestoy, P. R., Duff, I. S., Koster, J. \& L'Excellent, J.-Y. 2001 A fully asynchronous multifrontal solver using distributed dynamic scheduling. SIAM Journal on Matrix Analysis and Applications 23 (1), 15-41.

Badia, S., Quaini, A. \& QuARTeroni, A. 2009 Coupling Biot and Navier-Stokes equations for modelling fluid-poroelastic media interaction. Journal of Computational Physics 228 (21), 7986-8014.

Beavers, G. S. \& Joseph, D. D. 1967 Boundary conditions at a naturally permeable wall. Journal of Fluid Mechanics 30, 197-207.

Berkouk, K. K., Carpenter, P. W. \& Lucey, A. D. 2003 Pressure wave propagation in fluid-filled co-axial elastic tubes part 1: Basic theory. ASME Journal of Biomechanical Engineering 125, 852-856.

Bertram, C. D. 2009 A numerical investigation of waves propagating in the spinal cord and subarachnoid space in the presence of a syrinx. Journal of Fluids and Structures 25, 11891205.

Bertram, C. D. 2010 Evaluation by fluid/structure-interaction spinal-cord simulation of the effects of subarachnoid-space stenosis on an adjacent syrinx. ASME Journal of Biomechanical Engineering 132, 1-15. 
Bertram, C. D., Brodbelt, A. R. \& Stoodley, M. A. 2005 The origins of syringomyelia: numerical models of fluid/structure interactions in the spinal cord. ASME Journal of Biomechanical Engineering 127, 1099-1109.

Bertram, C. D. \& Heil, M. 2016 A poroelastic fluid/structure-interaction model of cerebrospinal fluid dynamics in the cord with syringomyelia and adjacent subarachnoid-space stenosis. ASME Journal of Biomechanical Engineering (in press).

Bunck, A. C., Kroger, J.-R., Juttner, A., Brentrup, A., Fiedler, B., Schaarschmidt, F., Crelier, G. R., Schwindt, W., Heindel, W., Niederstadt, T. \& Maintz, D. 2011 Magnetic resonance 4D flow characteristics of cerebrospinal fluid at the craniocervical junction and the cervical spinal canal. European Radiology 21, 1788-1796.

Carpenter, P. W., Berkouk, K. K. \& Lucey, A. D. 2003 Pressure wave propagation in fluid-filled co-axial elastic tubes part 2: Mechanisms for the pathogenesis of syringomyelia. ASME Journal of Biomechanical Engineering 125, 857-863.

Carraro, T., Goll, C., Marciniak-Czochra, A. \& Mikelic, A. 2013 Pressure jump interface law for the stokesdarcy coupling: confirmation by direct numerical simulations. Journal of Fluid Mechanics 732, 510-536.

Chang, G. L. \& Hung, T. K. 1988 An in-vivo measurement and analysis of viscoelastic properties of the spinal cord of cats. ASME Journal of Biomechanical Engineering 110, $115-122$.

Cirovic, S. 2009 A coaxial tube model of the cerebrospinal fluid pulse propagation in the spinal column. ASME Journal of Biomechanical Engineering 131, 021008-021001021009.

Cirovic, S. \& Kim, M. 2012 A one-dimensional model of the spinal cerebrospinal-fluid compartment. ASME Journal of Biomechanical Engineering 134, 021005-021001021010.

Cowin, S. C. 1999 Bone poroelasticity. Journal of Biomechanics 32 (3), 217-238.

Detournay, E. \& Cheng, A.H.-D. 1993 Fundamentals of poroelasticity. In Comprehensive Rock Engineering: Principles, Practice and Projects, Vol. II, Analysis and Design Method (ed. C. Fairhurst), pp. 113-171. Pergamon Press.

Dreha-Kulaczewsit, S., Joseph, A. A., Merboldt, K.-D., Ludwig, H.-C., Gärtner, J. \& Frahm, J. 2015 Inspiration is the major regulator of human CSF flow. The Journal of Neuroscience 35 (6), 2485-2491.

Elliott, N. J. 2012 Syrinx fluid transport: Modeling pressure-wave-induced flux across the spinal pial membrane. ASME Journal of Biomechanical Engineering 134, 031006-0310069.

Elliott, N. S. J., Bertram, C. D., Martin, B. A. \& Brodbelt, A. R. 2013 Syringomyelia: A review of the biomechanics. Journal of Fluids and Structures 40, 1-24.

Elliott, N. S. J., Lockerby, D. A. \& Brodbelt, A. R. 2009 The pathogenesis of syringomyelia: a re-evaluation of the elastic-jump hypothesis. ASME Journal of Biomechanical Engineering 131, 044503-044501044506.

Ervin, V. J. 2012 Computational bases for $R T_{k}$ and $B D M_{k}$ on triangles. Computers \& Mathematics with Applications 64, 2765-2774.

ERvin, V. J. 2013 Approximation of coupled Stokes-Darcy flow in an axisymmetric domain. Computer Methods in Applied Mechanics and Engineering 258, 96-108.

Gupta, S., Soellinger, M., Boesiger, P., Poulikakos, D. \& Kurtcuoglu, V. 2008 Threedimensional computational modeling of subject-specific cerebrospinal fluid flow in the subarachnoid space. ASME Journal of Biomechanical Engineering 131, 021010-021010-11.

Gupta, S., Soellinger, M., Grzybowski, D. M., Boesiger, P., Biddiscombe, J., Poulikakos, D. \& Kurtcuoglu, V. 2010 Cerebrospinal fluid dynamics in the human cranial subarachnoid space: an overlooked mediator of cerebral disease. i. computational model. Journal of the Royal Society Interface 7, 1195-1204.

Hazel, A. L., Heil, M., Waters, S. L. \& Oliver, J. M. 2012 On the liquid lining in fluidconveying curved tubes. Journal of Fluid Mechanics 705, 213-233.

Heidari Pahlavian, S., Loth, F., Luciano, M., Oshinski, J. \& Martin, B. A. 2015 Neural tissue motion impacts cerebrospinal fluid dynamics at the cervical medullary junction: A patient-specific moving-boundary computational model. Annals of biomedical engineering 43 (12), 2911-2923.

Heidari Pahlavian, S., Yiallourou, T., Tubbs, R. S., Bunck, A. C., Loth, F., Goodin, M., Raisee, M. \& Martin, B. A. 2014 The impact of spinal cord nerve roots and den- 
ticulate ligaments on cerebrospinal fluid dynamics in the cervical spine. PLoS ONE 9 (4), e91888.

Heil, M. \& HAzel, A. L. 2006 oomph-lib - an object-oriented multi-physics finite-element library. In Fluid-Structure Interaction (ed. M. Schäfer \& H.-J. Bungartz), pp. 19-49. Springer, oomph-lib is available as open-source software at http://www.oomph-lib.org.

Hentschel, S., Mardal, K.-A., Lovgren, A. E., Linge, S. \& Haughton, V. 2010 Characterization of cyclic CSF flow in the foramen magnum and upper cervical spinal canal with MR flow imaging and computational fluid dynamics. American Journal of Neuroradiology 31, 997-1002.

Hewitt, R. E., Hazel, A. L., Clarke, R. J. \& Denier, J. P. 2011 Unsteady flow in a torus after a sudden change in rotation rate. Journal of Fluid Mechanics 688, 88-119.

Humphreys, J. D. 2008 Mechanisms of arterial remodelling in hypertension: Coupled roles of wall shear and intramural stress. Hypertension 52 (2), 195-200.

Kistler, S. F. \& SCRiven, L. E. 1983 Coating flows. In Computational Analysis of Polymer Processing (ed. J.R.A. Pearson \& S.M. Richardson), pp. 243-299. London: Applied Science Publishers.

Linninger, A. A., Xenos, M., Zhu, D. C., Somayaji, M. R., Srinivasa Kondapalli, S. \& PENN, R. D. 2007 Cerebrospinal fluid flow in the normal and hydrocephalic human brain. IEEE Transactions on Biomedical Engineering 54 (2), 291-302.

Loth, Francis, Yardimci, M Atif \& Alperin, NoAm 2001 Hydrodynamic modeling of cerebrospinal fluid motion within the spinal cavity. Journal of biomechanical engineering 123 (1), 71-79.

Martin, B. A., Labuda, R., Royston, T. J., Oshinski, J. N., Iskandar, B. \& Loth, F. 2010 Spinal subarachnoid space pressure measurements in an in vitro spinal stenosis model: implications on syringomyelia theories. Journal of Biomechanical Engineering 132, $111007-111001111011$.

Pinler-Puzovic, D., Juel, A., Peng, G. G., Lister, J. R. \& Heil, M. 2015 Displacement flows under elastic membranes. Part 1. experiments and direct numerical simulations. Journal of Fluid Mechanics 784, 487-511.

Rossi, C., Boss, A., Steidle, G., Martirosian, P., Klose, U., Capuani, S., Maraviglia, B., Claussen, C. D. \& Schick, F. 2008 Water diffusion anisotropy in white and gray matter of the human spinal cord. Journal of Magnetic Resonance Imaging 27 (3), 476-482.

SAFFMAN, P. 1971 On the boundary condition at the surface of a porous medium. Studies in Applied Mathematics 50, 93-101.

Shaffer, Nicholas, Martin, Bryn \& Loth, Francis 2011 Cerebrospinal fluid hydrodynamics in type I chiari malformation. Neurological Research 33 (3), 247-260.

Shewchuk, J. R. 1996 Triangle: Engineering a 2D Quality Mesh Generator and Delaunay Triangulator. In Applied Computational Geometry: Towards Geometric Engineering (ed. Ming C. Lin \& Dinesh Manocha), Lecture Notes in Computer Science, vol. 1148, pp. 203222. Springer, from the First ACM Workshop on Applied Computational Geometry.

Simon, B. R. 1992 Multiphase poroelastic finite element models for soft tissue structure. Applied Mechanics Reviews 45 (6).

Smillie, A., Sobey, I. \& Molnar, Z. 2005 A hydroelastic model of hydrocephalus. Journal of Fluid Mechanics 539, 417-443.

Stockman, H. W. 2006 Effect of anatomical fine structure on the flow of cerebrospinal fluid in the spinal subarachnoid space. ASME Journal of Biomechanical Engineering 128, 106-114.

Støverud, K. H, Alnæs, M., Langtangen, H. P., Haughton, V. \& Mardal, K.-A. 2015 Poro-elastic modeling of syringomyelia-a systematic study of the effects of pia mater, central canal, median fissure, white and gray matter on pressure wave propagation and fluid movement within the cervical spinal cord. Computer methods in biomechanics and biomedical engineering pp. 1-13.

Tully, B. \& Ventikos, Y. 2011 Cerebral water transport using multiple-network poroelastic theory: application to normal pressure hydrocephalus. Journal of Fluid Mechanics 667, $188-215$.

VAn De Vosse, F. N. \& Stergiopulos, N. 2011 Pulse wave propagation in the arterial tree. Annual Review of Fluid Mechanics 43 (1), 467-499.

Yiallourou, T. I., Kröger, J. R., Stergiopulos, N., Maintz, D., Martin, B. A. \& 


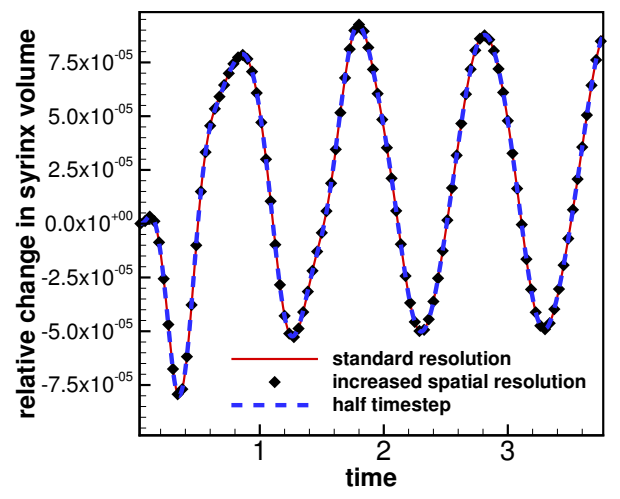

FiguRE 14. Evolution of the relative change in syrinx volume, $\Delta V_{\text {syrinx }}(t) / V_{\text {syrinx }}(t=0)$ for the standard resolution (red solid line); an increased temporal (thick blue dashed line) and spatial (markers) resolution, respectively. Parameters as in Fig. 6.

Bunck, A. C. 2015 Quantitative comparison of 4D MRI flow measurements to 3D computational fluid dynamics simulation of cerebrospinal fluid movement in the spinal subarachnoid space. PLoS ONE 7, e52284.

\section{Appendix A. Validation and spatio-temporal convergence tests}

oomph-lib's axisymmetric Navier-Stokes equations and FSI capabilities are well validated and have, over the years, been used in a wide variety of studies (e.g. Hewitt et al. 2011, Hazel et al. 2012, Pihler-Puzovic et al. 2015). To validate the driver code developed for the problem considered in this paper we initially re-computed some of the cases from Bertram's (2010) ADINA-based study of the impermeable system. Bertram (2010) performed these computations allowing for a realistic degree of liquid compressibility but the Mach number of the flows is so small that this had no practical effect on the results. The predictions for key quantities, such as the cycle-average fluid pressures shown in Fig. 14 in the present paper, agreed extremely well with Bertram's (2010) original results. The newly-implemented equations of poroelasticity were validated by comparing the numerical results against a variety of analytical solutions such as those presented in Fig. 10 in this paper. Fig. 14 shows the results of spatial and temporal convergence tests for the fully-coupled FSI simulations. The red solid line shows the relative change in syrinx volume for the standard spatial resolution (80 time-steps per period and a spatial discretisation with approximately 1.5 million degrees of freedom). This is compared to results from a simulation with twice the number of time-steps per period (thick blue dashed line), and with an increased spatial resolution (9.4 million degrees of freedom). The figure indicates that the results obtained with the standard spatial/temporal resolution are fully converged. 